\title{
Almost periodic solutions for neutral delay Hopfield neural networks with time-varying delays in the leakage term on time scales
}

\author{
Ling Li, Yongkun Li and Li Yang
}

Correspondence: yklie@ynu.edu.cn Department of Mathematics, Yunnan University, Kunming, Yunnan 650091, People's Republic of China

\begin{abstract}
In this paper, a class of neutral delay Hopfield neural networks with time-varying delays in the leakage term on time scales is considered. By utilizing the exponential dichotomy of linear dynamic equations on time scales, Banach's fixed point theorem and the theory of calculus on time scales, some sufficient conditions are obtained for the existence and exponential stability of almost periodic solutions for this class of neural networks. Finally, a numerical example illustrates the feasibility of our results and also shows that the continuous-time neural network and the discrete-time analogue have the same dynamical behaviors. The results of this paper are completely new and complementary to the previously known results even when the time scale $\mathbb{T}=\mathbb{R}$ or $\mathbb{Z}$.
\end{abstract}

Keywords: almost periodic solutions; Hopfield neural networks; neutral delay; leakage term; time scales

\section{Introduction}

The dynamical properties for delayed Hopfield neural networks have been extensively studied since they can be applied into pattern recognition, image processing, speed detection of moving objects, optimization problems and many other fields. Besides, due to the finite speed of information processing, the existence of time delays frequently causes oscillation, divergence, or instability in neural networks. Therefore, it is of prime importance to consider the delay effects on the stability of neural networks. Up to now, neural networks with various types of delay have been widely investigated by many authors [1-20].

However, so far, very little attention has been paid to neural networks with time delay in the leakage (or 'forgetting') term [21-35]. Such time delays in the leakage terms are difficult to handle and have been rarely considered in the literature. In fact, the leakage term has a great impact on the dynamical behavior of neural networks. Also, recently, another type of time delays, namely, neutral-type time delays which always appear in the study of automatic control, population dynamics and vibrating masses attached to an elastic bar, etc., has drawn much research attention. So far there have been only a few papers that have taken neutral-type phenomenon into account in delayed neural networks [33-43].

In fact, both continuous and discrete systems are very important in implementation and applications. But it is troublesome to study the existence of almost periodic solutions for continuous and discrete systems respectively. Therefore, it is meaningful to study that on time scales which can unify the continuous and discrete situations (see [44-50]).

( ) $2014 \mathrm{Li}$ et al.: licensee Springer. This is an Open Access article distributed under the terms of the Creative Commons Attribution License (http://creativecommons.org/licenses/by/2.0), which permits unrestricted use, distribution, and reproduction in any medium, provided the original work is properly cited. 
To the best of our knowledge, up to now, there have been no papers published on the existence and stability of almost periodic solutions to neutral-type delay neural networks with time-varying delays in the leakage term on time scales. Thus, it is important and, in effect, necessary to study the existence of almost periodic solutions for neutral-type neural networks with time-varying delay in the leakage term on time scales.

Motivated by above, in this paper, we propose the following neutral delay Hopfield neural networks with time-varying delays in the leakage term on time scale $\mathbb{T}$ :

$$
\begin{aligned}
x_{i}^{\Delta}(t)= & -c_{i}(t) x_{i}\left(t-\eta_{i}(t)\right)+\sum_{j=1}^{n} a_{i j}(t) f_{j}\left(x_{j}\left(t-\tau_{i j}(t)\right)\right) \\
& +\sum_{j=1}^{n} d_{i j}(t) \int_{t-\delta_{i j}(t)}^{t} \theta_{i j}(s) h_{j}\left(x_{j}(s)\right) \Delta s+\sum_{j=1}^{n} b_{i j}(t) g_{j}\left(x_{j}^{\Delta}\left(t-\sigma_{i j}(t)\right)\right) \\
& +\sum_{j=1}^{n} e_{i j}(t) \int_{t-\zeta_{i j}(t)}^{t} \xi_{i j}(s) k_{j}\left(x_{j}^{\Delta}(s)\right) \Delta s+I_{i}(t), \quad i=1,2, \ldots, n,
\end{aligned}
$$

where $\mathbb{T}$ is an almost periodic time scale that will be defined in the next section, $x_{i}(t)$ denotes the potential (or voltage) of cell $i$ at time $t, c_{i}(t)>0$ represents the rate at which the $i$ th unit will reset its potential to the resting state in isolation when disconnected from the network and external inputs at time $t, a_{i j}(t), b_{i j}(t), d_{i j}(t)$ and $e_{i j}(t)$ represent the delayed strengths of connectivity and neutral delayed strengths of connectivity between cell $i$ and $j$ at time $t$, respectively, $\theta_{i j}(\cdot)$ and $\xi_{i j}(\cdot)$ are the kernel functions determining the distributed delays, $f_{j}, g_{j}, h_{j}$ and $k_{j}$ are the activation functions in system (1.1), $I_{i}(t)$ is an external input on the $i$ th unit at time $t, \tau_{i j}(t) \geq 0$ and $\sigma_{i j}(t) \geq 0$ correspond to the transmission delays of the $i$ th unit along the axon of the $j$ th unit at time $t$.

If $\mathbb{T}=\mathbb{R}$, then system (1.1) is reduced to the following continuous-time neutral delay Hopfield neural network:

$$
\begin{aligned}
x_{i}^{\prime}(t)= & -c_{i}(t) x_{i}\left(t-\eta_{i}(t)\right)+\sum_{j=1}^{n} a_{i j}(t) f_{j}\left(x_{j}\left(t-\tau_{i j}(t)\right)\right) \\
& +\sum_{j=1}^{n} d_{i j}(t) \int_{t-\delta_{i j}(t)}^{t} \theta_{i j}(s) h_{j}\left(x_{j}(s)\right) \mathrm{d} s+\sum_{j=1}^{n} b_{i j}(t) g_{j}\left(x_{j}^{\prime}\left(t-\sigma_{i j}(t)\right)\right) \\
& +\sum_{j=1}^{n} e_{i j}(t) \int_{t-\zeta_{i j}(t)}^{t} \xi_{i j}(s) k_{j}\left(x_{j}^{\prime}(s)\right) \mathrm{d} s+I_{i}(t), \quad i=1,2, \ldots, n,
\end{aligned}
$$

and if $\mathbb{T}=\mathbb{Z}$, then system (1.1) is reduced to the discrete-time neutral delay Hopfield neural network

$$
\begin{aligned}
\Delta x_{i}(t)= & -c_{i}(t) x_{i}\left(t-\eta_{i}(t)\right)+\sum_{j=1}^{n} a_{i j}(t) f_{j}\left(x_{j}\left(t-\tau_{i j}(t)\right)\right) \\
& +\sum_{j=1}^{n} d_{i j}(t) \sum_{s=t-\delta_{i j}(t)}^{t} \theta_{i j}(s) h_{j}\left(x_{j}(s)\right)+\sum_{j=1}^{n} b_{i j}(t) g_{j}\left(\Delta x_{j}\left(t-\sigma_{i j}(t)\right)\right) \\
& +\sum_{j=1}^{n} e_{i j}(t) \sum_{s=t-\zeta_{i j}(t)}^{t} \xi_{i j}(s) k_{j}\left(\Delta x_{j}(s)\right)+I_{i}(t), \quad i=1,2, \ldots, n,
\end{aligned}
$$


where $t \in \mathbb{Z}$ and $\Delta x(t)=x(t+1)-x(t)$. When $\eta_{i}(t) \equiv 0, d_{i j}(t) \equiv 0, e_{i j}(t) \equiv 0, i, j=1,2, \ldots, n$, Bai [37] and Xiao [38] studied the almost periodicity of (1.2), respectively. However, even when $\eta_{i}(t) \equiv 0, i=1,2, \ldots, n$, the almost periodicity to (1.3), the discrete-time analogue of (1.2), has been not studied yet.

For convenience, for any almost periodic function $f(t)$ defined on $\mathbb{T}$, we define $f^{-}=$ $\inf _{i \in \mathbb{T}}|f(t)|, f^{+}=\sup _{\in \mathbb{T}}|f(t)|$.

The initial condition associated with system (1.1) is of the form

$$
x_{i}(t)=\varphi_{i}(t), \quad x_{i}^{\Delta}(t)=\varphi_{i}^{\Delta}(t), \quad t \in[-l, 0]_{\mathbb{T}}, i=1,2, \ldots, n,
$$

where $\varphi_{i}(\cdot)$ denotes a real-value bounded $\Delta$-differentiable function defined on $[-\iota, 0]_{\mathbb{T}}$ and $\iota=\max _{1 \leq i, j \leq n}\left\{\eta_{i}^{+}, \tau_{i j}^{+}, \sigma_{i j}^{+}, \delta_{i j}^{+}, \zeta_{i j}^{+}\right\}$.

Throughout this paper, we assume that:

$\left(\mathrm{H}_{1}\right) \quad c_{i}(t)>0$ with $-c_{i} \in \mathcal{R}^{+}, \eta_{i}(t) \geq 0, \tau_{i j}(t) \geq 0, \sigma_{i j}(t) \geq 0, \delta_{i j}(t) \geq 0, \zeta_{i j}(t) \geq 0, a_{i j}(t), b_{i j}(t)$, $d_{i j}(t), e_{i j}(t)$ and $I_{i}(t)$ are all almost periodic functions on $\mathbb{T}, t-\eta_{i}(t) \in \mathbb{T}, t-\tau_{i j}(t) \in \mathbb{T}$, $t-\sigma_{i j}(t) \in \mathbb{T}, t-\delta_{i j}(t) \in \mathbb{T}, t-\zeta_{i j}(t) \in \mathbb{T}$ for $t \in \mathbb{T}, i, j=1,2, \ldots, n$.

$\left(\mathrm{H}_{2}\right)$ There exist positive constants $L_{i}>0, l_{i}>0, L_{i}^{h}>0, l_{i}^{k}>0$ such that for $i=1,2, \ldots, n$,

$$
\begin{array}{lc}
\left|f_{i}(x)-f_{i}(y)\right| \leq L_{i}|x-y|, & \left|g_{i}(x)-g_{i}(y)\right| \leq l_{i}|x-y|, \\
\left|h_{i}(x)-h_{i}(y)\right| \leq L_{i}^{h}|x-y|, & \left|k_{i}(x)-k_{i}(y)\right| \leq l_{i}^{k}|x-y|,
\end{array}
$$

where $x, y \in \mathbb{R}$ and $f_{i}(0)=g_{i}(0)=h_{i}(0)=k_{i}(0)=0$.

$\left(\mathrm{H}_{3}\right)$ For $i, j=1,2, \ldots, n$, the delay kernels $\theta_{i j}, \xi_{i j}: \mathbb{T} \rightarrow \mathbb{R}$ are continuous and integrable with

$$
0 \leq \int_{t-\delta_{i j}^{+}}^{t}\left|\theta_{i j}(s)\right| \Delta s \leq \bar{\theta}_{i j}, \quad 0 \leq \int_{t-\zeta_{i j}^{+}}^{t}\left|\xi_{i j}(s)\right| \Delta s \leq \bar{\xi}_{i j} .
$$

Our main purpose of this paper is to study the existence and global exponential stability of the almost periodic solution to (1.1). Our results of this paper are completely new and complementary to the previously known results even when the time scale $\mathbb{T}=\mathbb{R}$ or $\mathbb{Z}$. The organization of the rest of this paper is as follows. In Section 2, we introduce some definitions and make some preparations for later sections. In Section 3 and Section 4, by utilizing Banach's fixed point theorem and the theory of calculus on time scales, we present some sufficient conditions which guarantee the existence of a unique globally exponentially stable almost periodic solution for system (1.1). In Section 5, we present examples to illustrate the feasibility and effectiveness of our results obtained in previous sections. We draw a conclusion in Section 6.

\section{Preliminaries}

In this section, we shall first recall some basic definitions and lemmas which will be useful for the proof of our main results.

Let $\mathbb{T}$ be a nonempty closed subset (time scale) of $\mathbb{R}$. The forward and backward jump operators $\sigma, \rho: \mathbb{T} \rightarrow \mathbb{T}$ and the graininess $\mu: \mathbb{T} \rightarrow \mathbb{R}^{+}$are defined, respectively, by

$$
\sigma(t)=\inf \{s \in \mathbb{T}: s>t\}, \quad \rho(t)=\sup \{s \in \mathbb{T}: s<t\} \quad \text { and } \quad \mu(t)=\sigma(t)-t .
$$


A point $t \in \mathbb{T}$ is called left-dense if $t>\inf \mathbb{T}$ and $\rho(t)=t$, left-scattered if $\rho(t)<t$, rightdense if $t<\sup \mathbb{T}$ and $\sigma(t)=t$, and right-scattered if $\sigma(t)>t$. If $\mathbb{T}$ has a left-scattered maximum $m$, then $\mathbb{T}^{k}=\mathbb{T} \backslash\{m\}$; otherwise $\mathbb{T}^{k}=\mathbb{T}$. If $\mathbb{T}$ has a right-scattered minimum $m$, then $\mathbb{T}_{k}=\mathbb{T} \backslash\{m\}$; otherwise $\mathbb{T}_{k}=\mathbb{T}$.

A function $f: \mathbb{T} \rightarrow \mathbb{R}$ is right-dense continuous provided it is continuous at right-dense point in $\mathbb{T}$ and its left-side limits exist at left-dense points in $\mathbb{T}$. If $f$ is continuous at each right-dense point and each left-dense point, then $f$ is said to be a continuous function on $\mathbb{T}$.

For $y: \mathbb{T} \rightarrow \mathbb{R}$ and $t \in \mathbb{T}^{k}$, we define the delta derivative of $y(t), y^{\Delta}(t)$, to be the number (if it exists) with the property that for a given $\varepsilon>0$, there exists a neighborhood $U$ of $t$ such that

$$
\left|[y(\sigma(t))-y(s)]-y^{\Delta}(t)[\sigma(t)-s]\right|<\varepsilon|\sigma(t)-s|
$$

for all $s \in U$.

If $y$ is continuous, then $y$ is right-dense continuous, and if $y$ is delta differentiable at $t$, then $y$ is continuous at $t$.

Let $y$ be right-dense continuous. If $Y^{\Delta}(t)=y(t)$, then we define the delta integral by

$$
\int_{a}^{t} y(s) \Delta s=Y(t)-Y(a)
$$

A function $r: \mathbb{T} \rightarrow \mathbb{R}$ is called regressive if

$$
1+\mu(t) r(t) \neq 0
$$

for all $t \in \mathbb{T}^{k}$. The set of all regressive and $r d$-continuous functions $r: \mathbb{T} \rightarrow \mathbb{R}$ will be denoted by $\mathcal{R}=\mathcal{R}(\mathbb{T})=\mathcal{R}(\mathbb{T}, \mathbb{R})$. We define the set $\mathcal{R}^{+}=\mathcal{R}^{+}(\mathbb{T}, \mathbb{R})=\{r \in \mathcal{R}: 1+\mu(t) r(t)>$ $0, \forall t \in \mathbb{T}\}$.

If $r$ is a regressive function, then the generalized exponential function $e_{r}$ is defined by

$$
e_{r}(t, s)=\exp \left\{\int_{s}^{t} \xi_{\mu(\tau)}(r(\tau)) \Delta \tau\right\} \quad \text { for } s, t \in \mathbb{T}
$$

with the cylinder transformation

$$
\xi_{h}(z)= \begin{cases}\frac{\log (1+h z)}{h} & \text { if } h \neq 0 \\ z & \text { if } h=0\end{cases}
$$

Let $p, q: \mathbb{T} \rightarrow \mathbb{R}$ be two regressive functions, we define

$$
p \oplus q:=p+q+\mu p q, \quad \ominus p:=-\frac{p}{1+\mu p}, \quad p \ominus q:=p \oplus(\ominus q)
$$

Then the generalized exponential function has the following properties.

Definition 2.1 [51] Let $p, q: \mathbb{T} \rightarrow \mathbb{R}$ be two regressive functions, define

$$
p \oplus q=p+q+\mu p q, \quad \ominus p=-\frac{p}{1+\mu p}, \quad p \ominus q=p \oplus(\ominus q)
$$


Lemma 2.1 [51] Assume that $p, q: \mathbb{T} \rightarrow \mathbb{R}$ are two regressive functions, then

(1) $e_{0}(t, s) \equiv 1$ and $e_{p}(t, t) \equiv 1$;

(2) $e_{p}(\sigma(t), s)=(1+\mu(t) p(t)) e_{p}(t, s)$;

(3) $e_{p}(t, s)=\frac{1}{e_{p}(s, t)}=e_{\ominus p}(s, t)$;

(4) $e_{p}(t, s) e_{p}(s, r)=e_{p}(t, r)$

(5) $\left(e_{\ominus p}(t, s)\right)^{\Delta}=(\ominus p)(t) e_{\ominus p}(t, s)$;

(6) if $a, b, c \in \mathbb{T}$, then $\int_{a}^{b} p(t) e_{p}(c, \sigma(t)) \Delta t=e_{p}(c, a)-e_{p}(c, b)$.

Definition 2.2 [51] Assume that $f: \mathbb{T} \rightarrow \mathbb{R}$ is a function and let $t \in \mathbb{T}^{k}$. Then we define $f^{\Delta}(t)$ to be the number (provided it exists) with the property that given any $\varepsilon>0$, there is a neighborhood $U$ of $t$ (i.e., $U=(t-\delta, t+\delta) \cap \mathbb{T}$ for some $\delta>0$ ) such that

$$
\left|[f(\sigma(t))-f(s)]-f^{\Delta}(t)[\sigma(t)-s]\right| \leq \varepsilon|\sigma(t)-s|
$$

for all $s \in U$. We call $f^{\Delta}(t)$ the delta (or Hilger) derivative of $f$ at $t$. Moreover, we say that $f$ is delta (or Hilger) differentiable (or, in short, differentiable) on $\mathbb{T}^{k}$ provided $f^{\Delta}(t)$ exists for all $t \in \mathbb{T}^{k}$. The function $f^{\Delta}: \mathbb{T}^{k} \rightarrow \mathbb{R}$ is then called the (delta) derivative of $f$ on $\mathbb{T}^{k}$.

Definition 2.3 [52] A time scale $\mathbb{T}$ is called an almost periodic time scale if

$$
\Pi:=\{\tau \in \mathbb{R}: t \pm \tau \in \mathbb{T}, \forall t \in \mathbb{T}\} \neq\{0\}
$$

Definition 2.4 [52] Let $\mathbb{T}$ be an almost periodic time scale. A function $f \in C\left(\mathbb{T}, \mathbb{E}^{n}\right)$ is called an almost periodic function if the $\varepsilon$-translation set of

$$
E(\varepsilon, f)=\{\tau \in \Pi:|f(t+\tau)-f(t)|<\varepsilon, \forall t \in \mathbb{T}\}
$$

is a relatively dense set in $\mathbb{T}$ for all $\varepsilon>0$; that is, for any given $\varepsilon>0$, there exists a constant $l(\varepsilon)>0$ such that each interval of length $l(\varepsilon)$ contains a $\tau(\varepsilon) \in E(\varepsilon, f)$ such that

$$
|f(t+\tau)-f(t)|<\varepsilon, \quad \forall t \in \mathbb{T} .
$$

$\tau$ is called the $\varepsilon$-translation number of $f$ and $l(\varepsilon)$ is called the inclusion length of $T(\varepsilon, f)$.

Definition 2.5 [52] Let $A(t)$ be an $n \times n$ rd-continuous matrix on $\mathbb{T}$, the linear system

$$
x^{\Delta}(t)=A(t) x(t), \quad t \in \mathbb{T}
$$

is said to admit an exponential dichotomy on $\mathbb{T}$ if there exist positive constants $k, \alpha$, projection $P$ and the fundamental solution matrix $X(t)$ of (2.1), satisfying

$$
\begin{aligned}
& \left\|X(t) P X^{-1}(\sigma(s))\right\|_{0} \leq k e_{\ominus \alpha}(t, \sigma(s)), \quad s, t \in \mathbb{T}, t \geq \sigma(s), \\
& \left\|X(t)(I-P) X^{-1}(\sigma(s))\right\|_{0} \leq k e_{\ominus \alpha}(\sigma(s), t), \quad s, t \in \mathbb{T}, t \leq \sigma(s),
\end{aligned}
$$

where $\|\cdot\|_{0}$ is a matrix norm on $\mathbb{T}$ (say, for example, if $A=\left(a_{i j}\right)_{n \times m}$, then we can take $\left.\|A\|_{0}=\left(\sum_{i=1}^{n} \sum_{j=1}^{m}\left|a_{i j}\right|^{2}\right)^{\frac{1}{2}}\right)$. 
Consider the following almost periodic system:

$$
x^{\Delta}(t)=A(t) x(t)+f(t), \quad t \in \mathbb{T},
$$

where $A(t)$ is an almost periodic matrix function, $f(t)$ is an almost periodic vector function.

Lemma 2.2 [52] If the linear system (2.1) admits exponential dichotomy, then system (2.2) has a unique almost periodic solution

$$
x(t)=\int_{-\infty}^{t} X(t) P X^{-1}(\sigma(s)) f(s) \Delta s-\int_{t}^{+\infty} X(t)(I-P) X^{-1}(\sigma(s)) f(s) \Delta s,
$$

where $X(t)$ is the fundamental solution matrix of (2.1).

Lemma 2.3 [53] Let $c_{i}(t)$ be an almost periodic function on $\mathbb{T}$, where $c_{i}(t)>0,-c_{i}(t) \in \mathcal{R}^{+}$, $i=1,2, \ldots, n, \forall t \in \mathbb{T}$ and $\min _{1 \leq i \leq n}\left\{\inf _{t \in \mathbb{T}} c_{i}(t)\right\}=\widetilde{m}>0$, then the linear system

$$
x^{\Delta}(t)=\operatorname{diag}\left(-c_{1}(t),-c_{2}(t), \ldots,-c_{n}(t)\right) x(t)
$$

admits an exponential dichotomy on $\mathbb{T}$.

One can easily prove the following.

Lemma 2.4 Suppose that $f(t)$ is an rd-continuous function and $c(t)$ is a positive $r d$ continuous function satisfying $-c(t) \in \mathcal{R}^{+}$. Let

$$
g(t)=\int_{t_{0}}^{t} e_{-c}(t, \sigma(s)) f(s) \Delta s
$$

where $t_{0} \in \mathbb{T}$, then

$$
g^{\Delta}(t)=f(t)+\int_{t_{0}}^{t}\left[-c(t) e_{-c}(t, \sigma(s)) f(s)\right] \Delta s .
$$

\section{Existence of almost periodic solutions}

Let $A P(\mathbb{T})=\{x(t) \in C(\mathbb{T}, \mathbb{R}): x(t)$ is a real-valued, almost periodic function on $\mathbb{T}\}, \mathbb{Y}=$ $\left\{x(t) \in C^{1}(\mathbb{T}, \mathbb{R}): x(t), x^{\Delta}(t) \in A P(\mathbb{T})\right\}$ and

$$
\mathbb{X}=\left\{\varphi=\left(\varphi_{1}(t), \varphi_{2}(t), \ldots, \varphi_{n}(t)\right)^{T}: \varphi_{i}(t) \in \mathbb{Y}, i=1,2, \ldots, n\right\} .
$$

For $\forall \varphi \in \mathbb{X}$, if we define induced modulus $\|\varphi\|_{\mathbb{X}}=\max \left\{\|\varphi\|_{0},\left\|\varphi^{\Delta}\right\|_{0}\right\}$, where

$$
\|\varphi\|_{0}=\sup _{t \in \mathbb{T}}\|\varphi(t)\|_{0}, \quad\|\varphi(t)\|_{0}=\max _{1 \leq i \leq n}\left|\varphi_{i}(t)\right|
$$

and $\varphi^{\Delta}(t)=\left(\varphi_{1}^{\Delta}(t), \varphi_{2}^{\Delta}(t), \ldots, \varphi_{n}^{\Delta}(t)\right)^{T}$, then $\mathbb{X}$ is a Banach space.

Theorem 3.1 Assume that $\left(\mathrm{H}_{1}\right)-\left(\mathrm{H}_{3}\right)$ and

$\left(\mathrm{H}_{4}\right) \quad r=\max _{1 \leq i \leq n} \max \left\{\frac{1}{c_{i}^{-}}, 1+\frac{c_{i}^{+}}{c_{i}^{-}}\right\}\left(c_{i}^{+} \eta_{i}^{+}+\sum_{j=1}^{n} a_{i j}^{+} L_{j}+\sum_{j=i}^{n} b_{i j}^{+} l_{j}+\sum_{j=1}^{n} d_{i j}^{+} L_{j}^{h} \bar{\theta}_{i j}+\sum_{j=1}^{n} e_{i j}^{+} l_{j}^{k} \times\right.$ $\left.\bar{\xi}_{i j}\right)<1$ 
hold, then there exists exactly one almost periodic solution of system (1.1) in the region $\mathbb{X}_{0}=\left\{\phi(t) \mid\left\|\phi-\phi_{0}\right\|_{\mathbb{X}} \leq \frac{r R}{1-r}\right\}$, where

$$
\begin{aligned}
& R=\max _{1 \leq i \leq n} \max \left\{\frac{I_{i}^{+}}{c_{i}^{-}}, I_{i}^{+}\left(1+\frac{c_{i}^{+}}{c_{i}^{-}}\right)\right\}, \\
& \phi_{0}=\left(\int_{-\infty}^{t} I_{1}(s) e_{-c_{1}}(t, \sigma(s)) \Delta s, \ldots, \int_{-\infty}^{t} I_{n}(s) e_{-c_{n}}(t, \sigma(s)) \Delta s\right)^{T} .
\end{aligned}
$$

Proof Rewrite (1.1) in the form

$$
\begin{aligned}
x_{i}^{\Delta}(t)= & -c_{i}(t) x_{i}(t)+c_{i}(t) \int_{t-\eta_{i}(t)}^{t} x_{i}^{\Delta}(s) \Delta s+\sum_{j=1}^{n} a_{i j}(t) f_{j}\left(x_{j}\left(t-\tau_{i j}(t)\right)\right) \\
& +\sum_{j=1}^{n} d_{i j}(t) \int_{t-\delta_{i j}(t)}^{t} \theta_{i j}(s) h_{j}\left(x_{j}(s)\right) \Delta s+\sum_{j=1}^{n} b_{i j}(t) g_{j}\left(x_{j}^{\Delta}\left(t-\sigma_{i j}(t)\right)\right) \\
& +\sum_{j=1}^{n} e_{i j}(t) \int_{t-\zeta_{i j}(t)}^{t} \xi_{i j}(s) k_{j}\left(x_{j}^{\Delta}(s)\right) \Delta s+I_{i}(t), \quad i=1,2, \ldots, n .
\end{aligned}
$$

For any $\phi \in \mathbb{X}$, we consider the following system:

$$
\begin{aligned}
x_{i}^{\Delta}(t)= & -c_{i}(t) x_{i}(t)+c_{i}(t) \int_{t-\eta_{i}(t)}^{t} \phi_{i}^{\Delta}(s) \Delta s+\sum_{j=1}^{n} a_{i j}(t) f_{j}\left(\phi_{j}\left(t-\tau_{i j}(t)\right)\right) \\
& +\sum_{j=1}^{n} d_{i j}(t) \int_{t-\delta_{i j}(t)}^{t} \theta_{i j}(s) h_{j}\left(\phi_{j}(s)\right) \Delta s+\sum_{j=1}^{n} b_{i j}(t) g_{j}\left(\phi_{j}^{\Delta}\left(t-\sigma_{i j}(t)\right)\right) \\
& +\sum_{j=1}^{n} e_{i j}(t) \int_{t-\zeta_{i j}(t)}^{t} \xi_{i j}(s) k_{j}\left(\phi_{j}^{\Delta}(s)\right) \Delta s+I_{i}(t), \quad i=1,2, \ldots, n .
\end{aligned}
$$

Since $\min _{1 \leq i \leq n}\left\{\inf _{t \in \mathbb{T}} c_{i}(t)\right\}>0$, it follows from Lemma 2.2 and Lemma 2.3 that system (3.1) has a unique almost periodic solution which can be expressed as follows:

$$
x^{\phi}(t)=\left(x_{1}^{\phi}(t), x_{2}^{\phi}(t), \ldots, x_{n}^{\phi}(t)\right)^{T},
$$

where

$$
\begin{aligned}
x_{i}^{\phi}(t)= & \int_{-\infty}^{t} e_{-c_{i}}(t, \sigma(s))\left[c_{i}(s) \int_{s-\eta_{i}(s)}^{s} \phi_{i}^{\Delta}(u) \Delta u+\sum_{j=1}^{n} a_{i j}(s) f_{j}\left(\phi_{j}\left(s-\tau_{i j}(s)\right)\right)\right. \\
& +\sum_{j=1}^{n} d_{i j}(s) \int_{s-\delta_{i j}(s)}^{s} \theta_{i j}(u) h_{j}\left(\phi_{j}(u)\right) \Delta u+\sum_{j=1}^{n} b_{i j}(s) g_{j}\left(\phi_{j}^{\Delta}\left(s-\sigma_{i j}(s)\right)\right) \\
& +\sum_{j=1}^{n} e_{i j}(s) \int_{s-\zeta i j}^{s}(s)
\end{aligned}
$$

Now, we define a mapping $T: \mathbb{X}_{0} \rightarrow \mathbb{X}_{0}$ by $(T \phi)(t)=x^{\phi}(t), \forall \phi \in \mathbb{X}_{0}$. 
By the definition of $\|\cdot\|_{\mathbb{X}}$, we have

$$
\begin{aligned}
\left\|\phi_{0}\right\|_{\mathbb{X}}= & \max \left\{\left\|\phi_{0}\right\|_{0},\left\|\phi_{0}^{\Delta}\right\|_{0}\right\} \\
= & \max \left\{\sup _{t \in \mathbb{T}} \max _{1 \leq i \leq n}\left|\int_{-\infty}^{t} I_{i}(s) e_{-c_{i}}(t, \sigma(s)) \Delta s\right|,\right. \\
& \left.\sup _{t \in \mathbb{T}} \max _{1 \leq i \leq n}\left|I_{i}(t)-\int_{-\infty}^{t} c_{i}(t) I_{i}(s) e_{-c_{i}}(t, \sigma(s)) \Delta s\right|\right\} \\
\leq & \max \left\{\max _{1 \leq i \leq n}\left\{\frac{I_{i}^{+}}{c_{i}^{-}}\right\}, \max _{1 \leq i \leq n}\left\{I_{i}^{+}\left(1+\frac{c_{i}^{+}}{c_{i}^{-}}\right)\right\}\right\}=R .
\end{aligned}
$$

Hence, for any $\phi \in \mathbb{X}_{0}=\left\{\phi \mid \phi \in \mathbb{X},\left\|\phi-\phi_{0}\right\|_{\mathbb{X}} \leq \frac{r R}{1-r}\right\}$, one has

$$
\|\phi\|_{\mathbb{X}} \leq\left\|\phi_{0}\right\|_{\mathbb{X}}+\left\|\phi-\phi_{0}\right\|_{\mathbb{X}} \leq R+\frac{r R}{1-r}=\frac{R}{1-r}
$$

Next, we will show that $T\left(\mathbb{X}_{0}\right) \subset \mathbb{X}_{0}$. In fact, for any $\phi \in \mathbb{X}_{0}$, we have

$$
\begin{aligned}
\| T \phi & \phi_{0} \|_{0} \\
= & \sup _{t \in \mathbb{T}} \max _{1 \leq i \leq n}\left\{\mid \int_{-\infty}^{t} e_{-c_{i}}(t, \sigma(s))\left[c_{i}(s) \int_{s-\eta_{i}(s)}^{s} \phi_{i}^{\Delta}(u) \Delta u\right.\right. \\
& +\sum_{j=1}^{n} d_{i j}(s) \int_{s-\delta_{i j}(s)}^{s} \theta_{i j}(u) h_{j}\left(\phi_{j}(u)\right) \Delta u+\sum_{j=1}^{n} b_{i j}(s) g_{j}\left(\phi_{j}^{\Delta}\left(s-\sigma_{i j}(s)\right)\right) \\
& \left.\left.+\sum_{j=1}^{n} e_{i j}(s) \int_{s-\zeta_{i j}(s)}^{s} \xi_{i j}(u) k_{j}\left(\phi_{j}^{\Delta}(u)\right) \Delta u\right] \Delta s \mid\right\} \\
\leq & \sup _{t \in \mathbb{T}} \max _{1 \leq i \leq n}\left\{\int _ { - \infty } ^ { t } e _ { - c _ { i } } ( t , \sigma ( s ) ) \left[c_{i}^{+}\left\|\phi^{\Delta}\right\|_{0} \eta_{i}^{+}+\sum_{j=1}^{n} a_{i j}^{+} L_{j}\|\phi\|_{0}+\sum_{j=1}^{n} b_{i j}^{+} l_{j}\left\|\phi^{\Delta}\right\|_{0}\right.\right. \\
& \left.\left.+\sum_{j=1}^{n} d_{i j}^{+} L_{j}^{h} \bar{\theta}_{i j}\|\phi\|_{0}+\sum_{j=1}^{n} e_{i j}^{+} l_{j}^{k} \bar{\xi}_{i j}\left\|\phi^{\Delta}\right\|_{0}\right] \Delta s\right\} \\
\leq & \max _{1 \leq i \leq n}\left\{\frac{1}{c_{i}^{-}}\left(c_{i}^{+} \eta_{i}^{+}+\sum_{j=1}^{n} a_{i j}^{+} L_{j}+\sum_{j=1}^{n} b_{i j}^{+} l_{j}+\sum_{j=1}^{n} d_{i j}^{+} L_{j}^{h} \bar{\theta}_{i j}+\sum_{j=1}^{n} e_{i j}^{+} l_{j}^{k} \bar{\xi}_{i j}\right)\right\}\|\phi\|_{\mathbb{X}} \\
& +\sum_{j=1}^{n} \max _{1 \leq i \leq n}\left\{\int _ { - \infty } ^ { t } e _ { - c _ { i } } ( t , \sigma ( s ) ) \left(c_{i}^{+} \eta_{i}^{+}+\sum_{j=1}^{h} \bar{\theta}_{i j}+\sum_{i j}^{+} L_{j}+\sum_{j=1}^{n} b_{i j}^{+} l_{j}\right.\right.
\end{aligned}
$$

and

$$
\begin{aligned}
& \left\|\left(T \phi-\phi_{0}\right)^{\Delta}\right\|_{0} \\
& \quad=\sup _{t \in \mathbb{T}} \max _{1 \leq i \leq n}\left\{\mid c_{i}(t) \int_{t-\eta_{i}(t)}^{t} \phi^{\Delta}(u) \Delta u+\sum_{j=1}^{n} a_{i j}(t) f_{j}\left(\phi_{j}\left(t-\tau_{i j}(t)\right)\right)\right.
\end{aligned}
$$


Li et al. Advances in Difference Equations 2014, 2014:178

Page 9 of 22

http://www.advancesindifferenceequations.com/content/2014/1/178

$$
\begin{aligned}
& +\sum_{j=1}^{n} d_{i j}(t) \int_{t-\delta_{i j}(t)}^{t} \theta_{i j}(s) h_{j}\left(x_{j}(s)\right) \Delta s+\sum_{j=1}^{n} b_{i j}(t) g_{j}\left(x_{j}^{\Delta}\left(t-\sigma_{i j}(t)\right)\right) \\
& +\sum_{j=1}^{n} e_{i j}(t) \int_{t-\zeta_{i j}(t)}^{t} \xi_{i j}(s) k_{j}\left(x_{j}^{\Delta}(s)\right) \Delta s \\
& +\int_{-\infty}^{t} c_{i}(t) e_{-c_{i}}(t, \sigma(s))\left[c_{i}(s) \int_{s-\eta_{i}(s)}^{s} \phi_{i}^{\Delta}(u) \Delta u\right. \\
& +\sum_{j=1}^{n} a_{i j}(s) f_{j}\left(\phi_{j}\left(s-\tau_{i j}(s)\right)\right)+\sum_{j=1}^{n} d_{i j}(s) \int_{s-\delta_{i j}(s)}^{s} \theta_{i j}(u) h_{j}\left(\phi_{j}(u)\right) \Delta u \\
& \left.\left.+\sum_{j=1}^{n} b_{i j}(s) g_{j}\left(\phi_{j}^{\Delta}\left(s-\sigma_{i j}(s)\right)\right)+\sum_{j=1}^{n} e_{i j}(s) \int_{s-\zeta_{i j}(s)}^{s} \xi_{i j}(u) k_{j}\left(\phi_{j}^{\Delta}(u)\right) \Delta u\right] \Delta s \mid\right\} \\
& \leq \sup _{t \in \mathbb{T}} \max _{1 \leq i \leq n}\left\{\left(c_{i}^{+} \eta_{i}^{+}+\sum_{j=1}^{n} a_{i j}^{+} L_{j}+\sum_{j=1}^{n} b_{i j}^{+} l_{j}+\sum_{j=1}^{n} d_{i j}^{+} L_{j}^{h} \bar{\theta}_{i j}+\sum_{j=1}^{n} e_{i j}^{+} l_{j}^{k} \bar{\xi}_{i j}\right)\|\phi\|_{X}\right. \\
& +c_{i}^{+} \int_{-\infty}^{t} e_{-c_{i}}(t, \sigma(s))\left(c_{i}^{+} \eta_{i}^{+}+\sum_{j=1}^{n} a_{i j}^{+} L_{j}+\sum_{j=1}^{n} b_{i j}^{+} l_{j}\right. \\
& \left.\left.+\sum_{j=1}^{n} d_{i j}^{+} L_{j}^{h} \bar{\theta}_{i j}+\sum_{j=1}^{n} e_{i j}^{+} j_{j}^{k} \bar{\xi}_{i j}\right)\|\phi\|_{X} \Delta s\right\} \\
& \leq \max _{1 \leq i \leq n}\left\{\left(1+\frac{c_{i}^{+}}{c_{i}^{-}}\right)\left(c_{i}^{+} \eta_{i}^{+}+\sum_{j=1}^{n} a_{i j}^{+} L_{j}+\sum_{j=1}^{n} b_{i j}^{+} l_{j}+\sum_{j=1}^{n} d_{i j}^{+} L_{j}^{h} \bar{\theta}_{i j}+\sum_{j=1}^{n} e_{i j}^{+} l_{j} \bar{\xi}_{j i j}\right)\right\}\|\phi\|_{X} .
\end{aligned}
$$

Thus, we obtain

$$
\begin{aligned}
& \left\|T \phi-\phi_{0}\right\|_{\mathbb{X}} \\
& =\max \left\{\left\|T \phi-\phi_{0}\right\|_{0},\left\|\left(T \phi-\phi_{0}\right)^{\Delta}\right\|_{0}\right\} \\
& \leq \max _{1 \leq i \leq n} \max \left\{\frac{1}{c_{i}^{-}}, 1+\frac{c_{i}^{+}}{c_{i}^{-}}\right\} \\
& \quad \times\left(c_{i}^{+} \eta_{i}^{+}+\sum_{j=1}^{n} a_{i j}^{+} L_{j}+\sum_{j=1}^{n} b_{i j}^{+} l_{j}+\sum_{j=1}^{n} d_{i j}^{+} L_{j}^{h} \bar{\theta}_{i j}+\sum_{j=1}^{n} e_{i j}^{+} l_{j}^{k} \bar{\xi}_{i j}\right)\|\phi\|_{\mathbb{X}} \\
& =r\|\phi\|_{\mathbb{X}} \leq \frac{r R}{1-r},
\end{aligned}
$$

which implies $(T \phi) \in \mathbb{X}_{0}$, so the mapping $T$ is a self-mapping from $\mathbb{X}_{0}$ to $\mathbb{X}_{0}$.

Finally, we prove that $T$ is a contraction mapping. Taking $\phi, \psi \in \mathbb{X}_{0}$, we have that

$$
\begin{aligned}
& \|T \phi-T \psi\|_{0} \\
& \quad \leq \max _{1 \leq i \leq n}\left\{\frac{1}{c_{i}^{-}}\left(c_{i}^{+} \eta_{i}^{+}+\sum_{j=1}^{n} a_{i j}^{+} L_{j}+\sum_{j=1}^{n} b_{i j}^{+} l_{j}+\sum_{j=1}^{n} d_{i j}^{+} L_{j}^{h} \bar{\theta}_{i j}+\sum_{j=1}^{n} e_{i j}^{+} l_{j}^{k} \bar{\xi}_{i j}\right)\right\}\|\phi-\psi\|_{\mathbb{X}} \\
& \leq r\|\phi-\psi\|_{\mathbb{X}}
\end{aligned}
$$


and

$$
\begin{aligned}
& \left\|(T \phi-T \psi)^{\Delta}\right\|_{0} \\
& \leq \max _{1 \leq i \leq n}\left\{\left(1+\frac{c_{i}^{+}}{c_{i}^{-}}\right)\left(c_{i}^{+} \eta_{i}^{+}+\sum_{j=1}^{n} a_{i j}^{+} L_{j}+\sum_{j=1}^{n} b_{i j}^{+} l_{j}+\sum_{j=1}^{n} d_{i j}^{+} L_{j}^{h} \bar{\theta}_{i j}+\sum_{j=1}^{n} e_{i j}^{+} l_{j}^{k} \bar{\xi}_{i j}\right)\right\} \\
& \quad \times\|\phi-\psi\|_{\mathbb{X}} \\
& \leq r\|\phi-\psi\|_{\mathbb{X}} .
\end{aligned}
$$

Noticing that $r<1$, it means that $T$ is a contraction mapping. Thus, there exists a unique fixed point $\phi \in \mathbb{X}_{0}$ such that $T \phi=\phi$. Then system (1.1) has a unique almost periodic solution in the region $\mathbb{X}_{0}=\left\{\phi(t) \in \mathbb{X} \mid\left\|\phi-\phi_{0}\right\| \leq \frac{r R}{1-r}\right\}$. This completes the proof.

\section{Exponential stability of the almost periodic solution}

Definition 4.1 The almost periodic solution $\bar{x}(t)=\left(\bar{x}_{1}(t), \bar{x}_{2}(t), \ldots, \bar{x}_{n}(t)\right)^{T}$ of system (1.1) with initial value $\bar{\varphi}(t)=\left(\bar{\varphi}_{1}(t), \bar{\varphi}_{2}(t), \ldots, \bar{\varphi}_{n}(t)\right)^{T}$ is said to be globally exponentially stable. If there exist positive constants $\lambda$ with $\ominus \lambda \in \mathcal{R}^{+}$and $M>1$ such that every solution $x(t)=\left(x_{1}(t), x_{2}(t), \ldots, x_{n}(t)\right)^{T}$ of system (1.1) with initial value $\varphi(t)=\left(\varphi_{1}(t), \varphi_{2}(t), \ldots, \varphi_{n}(t)\right)^{T}$ satisfies

$$
\|x(t)-\bar{x}(t)\|_{1} \leq M e_{\ominus \lambda}\left(t, t_{0}\right)\|\psi\|_{\mathbb{X}}, \quad \forall t \in[-\iota,+\infty)_{\mathbb{T}}, t \geq t_{0}
$$

where

$$
\begin{aligned}
& \|x(t)-\bar{x}(t)\|_{1}=\max \left\{\|x(t)-\bar{x}(t)\|_{0},\left\|(x(t)-\bar{x}(t))^{\Delta}\right\|_{0}\right\}, \\
& \|\psi\|_{X}=\max \left\{\sup _{t \in[-l, 0]_{\mathbb{T}}} \max _{1 \leq i \leq n}\left|\varphi_{i}(t)-\bar{\varphi}_{i}(t)\right|, \sup _{t \in[-l, 0]_{\mathbb{T}}} \max _{1 \leq i \leq n}\left|\varphi_{i}^{\Delta}(t)-\left(\bar{\varphi}_{i}\right)^{\Delta}(t)\right|\right\},
\end{aligned}
$$

and $t_{0} \in[-\iota, 0]_{\mathbb{T}}$.

Theorem 4.1 Assume that $\left(\mathrm{H}_{1}\right)-\left(\mathrm{H}_{4}\right)$ hold, then system (1.1) has a unique almost periodic solution which is globally exponentially stable.

Proof From Theorem 3.1, we see that system (1.1) has at least one almost periodic solution $\bar{x}(t)=\left(\bar{x}_{1}(t), \bar{x}_{2}(t), \ldots, \bar{x}_{n}(t)\right)^{T}$. Suppose that $x(t)=\left(x_{1}(t), x_{2}(t), \ldots, x_{n}(t)\right)^{T}$ is an arbitrary solution. Set $y_{i}(t)=x_{i}(t)-\bar{x}_{i}(t), i=1,2, \ldots, n$, then it follows from system (1.1) that

$$
\begin{aligned}
y_{i}^{\Delta}(t)= & x_{i}^{\Delta}(t)-\bar{x}_{i}^{\Delta}(t) \\
= & -c_{i}(t) x_{i}\left(t-\eta_{i}(t)\right)+\sum_{j=1}^{n} a_{i j}(t) f_{j}\left(x_{j}\left(t-\tau_{i j}(t)\right)\right) \\
& +\sum_{j=1}^{n} d_{i j}(t) \int_{t-\delta_{i j}(t)}^{t} \theta_{i j}(s) h_{j}\left(x_{j}(s)\right) \Delta s+\sum_{j=1}^{n} b_{i j}(t) g_{j}\left(x_{j}^{\Delta}\left(t-\sigma_{i j}(t)\right)\right) \\
& +\sum_{j=1}^{n} e_{i j}(t) \int_{t-\zeta_{i j}(t)}^{t} \xi_{i j}(s) k_{j}\left(x_{j}^{\Delta}(s)\right) \Delta s+c_{i}(t) \bar{x}_{i}\left(t-\eta_{i}(t)\right)
\end{aligned}
$$




$$
\begin{aligned}
& -\sum_{j=1}^{n} a_{i j}(t) f_{j}\left(\bar{x}_{j}\left(t-\tau_{i j}(t)\right)\right)-\sum_{j=1}^{n} b_{i j}(t) g_{j}\left(\bar{x}_{j}^{\Delta}\left(t-\sigma_{i j}(t)\right)\right) \\
& -\sum_{j=1}^{n} d_{i j}(t) \int_{t-\delta_{i j}(t)}^{t} \theta_{i j}(s) h_{j}\left(\bar{x}_{j}(s)\right) \Delta s-\sum_{j=1}^{n} e_{i j}(t) \int_{t-\zeta i j}^{t} \xi_{i j}(s) k_{j}\left(\bar{x}_{j}^{\Delta}(s)\right) \Delta s \\
= & -c_{i}(t) y_{i}\left(t-\eta_{i}(t)\right)+\sum_{j=1}^{n} a_{i j}(t)\left[f_{j}\left(x_{j}\left(t-\tau_{i j}(t)\right)\right)-f_{j}\left(\bar{x}_{j}\left(t-\tau_{i j}(t)\right)\right)\right] \\
& +\sum_{j=1}^{n} b_{i j}(t)\left[g_{j}\left(x_{j}^{\Delta}\left(t-\sigma_{i j}(t)\right)\right)-g_{j}\left(\bar{x}_{j}^{\Delta}\left(t-\sigma_{i j}(t)\right)\right)\right] \\
& +\sum_{j=1}^{n} d_{i j}(t) \int_{t-\delta_{i j}(t)}^{t} \theta_{i j}(s)\left[h_{j}\left(x_{j}(s)\right)-h_{j}\left(\bar{x}_{j}(s)\right)\right] \Delta s \\
& +\sum_{j=1}^{n} e_{i j}(t) \int_{t-\zeta_{i j}(t)}^{t} \xi_{i j}(s)\left[k_{j}\left(x_{j}^{\Delta}(s)\right)-k_{j}\left(\bar{x}_{j}^{\Delta}(s)\right)\right] \Delta s \\
= & -c_{i}(t) y_{i}\left(t-\eta_{i}(t)\right)+\sum_{j=1}^{n} a_{i j}(t) F_{j}\left(y_{j}\left(t-\tau_{i j}(t)\right)\right) \\
& +\sum_{j=1}^{n} b_{i j}(t) G_{j}\left(y_{j}^{\Delta}\left(t-\sigma_{i j}(t)\right)\right)+\sum_{j=1}^{n} d_{i j}(t) \int_{t-\delta_{i j}(t)}^{t} \theta_{i j}(s) H_{j}\left(y_{j}(s)\right) \Delta s \\
& +\sum_{j=1}^{n} e_{i j}(t) \int_{t-\zeta_{i j}(t)}^{t} \xi_{i j}(s) K_{j}\left(y_{j}^{\Delta}(s)\right) \Delta s, \\
&
\end{aligned}
$$

where $i=1,2, \ldots, n$ and for $i, j=1,2, \ldots, n$,

$$
\begin{aligned}
& F_{j}\left(y_{j}\left(t-\tau_{i j}(t)\right)\right)=f_{j}\left(y_{j}\left(t-\tau_{i j}(t)\right)+\bar{x}_{j}\left(t-\tau_{i j}(t)\right)\right)-f_{j}\left(\bar{x}_{j}\left(t-\tau_{i j}(t)\right)\right), \\
& g_{j}\left(y_{j}^{\Delta}\left(t-\sigma_{i j}(t)\right)\right)=g_{j}\left(y_{j}^{\Delta}\left(t-\sigma_{i j}(t)\right)+\bar{x}_{j}^{\Delta}\left(t-\sigma_{i j}(t)\right)\right)-g_{j}\left(\bar{x}_{j}^{\Delta}\left(t-\sigma_{i j}(t)\right)\right), \\
& H_{j}\left(y_{j}(s)\right)=h_{j}\left(x_{j}(s)\right)-h_{j}\left(\bar{x}_{j}(s)\right), \quad K_{j}\left(y_{j}^{\Delta}(s)\right)=k_{j}\left(x_{j}^{\Delta}(s)\right)-k_{j}\left(\bar{x}_{j}^{\Delta}(s)\right) .
\end{aligned}
$$

From $\left(\mathrm{H}_{2}\right)$ we have that for $i, j=1,2, \ldots, n$,

$$
\begin{aligned}
& \left|F_{j}\left(y_{j}\left(t-\tau_{i j}(t)\right)\right)\right| \leq L_{j}\left|y_{j}\left(t-\tau_{i j}(t)\right)\right|, \\
& \left|G_{j}\left(y_{j}^{\Delta}\left(t-\sigma_{i j}(t)\right)\right)\right| \leq l_{j}\left|y_{j}^{\Delta}\left(t-\sigma_{i j}(t)\right)\right|
\end{aligned}
$$

and

$$
\begin{aligned}
& \left|H_{j}\left(y_{j}(s)\right)\right| \leq L_{j}^{h}\left|y_{j}(s)\right|, \\
& \left|K_{j}\left(y_{j}^{\Delta}(s)\right)\right| \leq l_{j}^{k}\left|y_{j}^{\Delta}(s)\right| .
\end{aligned}
$$

The initial condition of (4.1) is

$$
\psi_{i}(t)=\varphi_{i}(t)-\bar{\varphi}_{i}(t), \quad \psi_{i}^{\Delta}(t)=\varphi_{i}^{\Delta}(t)-\bar{\varphi}_{i}^{\Delta}(t), \quad t \in[-l, 0]_{\mathbb{T}}, i=1,2, \ldots, n .
$$


Let $\Theta_{i}$ and $\Lambda_{i}$ be defined by

$$
\begin{aligned}
\Theta_{i}(\omega)= & c_{i}^{-}-\omega-\exp \left(\omega \sup _{s \in \mathbb{T}} \mu(s)\right)\left(c_{i}^{+} \eta_{i}^{+} \exp \left(\omega \eta_{i}^{+}\right)\right. \\
& +\sum_{j=1}^{n} a_{i j}^{+} L_{j} \exp \left(\omega \tau_{i j}^{+}\right)+\sum_{j=1}^{n} b_{i j}^{+} l_{j} \exp \left(\omega \sigma_{i j}^{+}\right) \\
& \left.+\sum_{j=1}^{n} d_{i j}^{+} L_{j}^{h} \bar{\theta}_{i j} \exp (\omega)+\sum_{j=1}^{n} e_{i j}^{+} l_{j}^{k} \bar{\xi}_{i j} \exp (\omega)\right), \quad i=1,2, \ldots, n
\end{aligned}
$$

and

$$
\begin{aligned}
\Lambda_{i}(\omega)= & c_{i}^{-}-\omega-\left(c_{i}^{+} \exp \left(\omega \sup _{s \in \mathbb{T}} \mu(s)\right)+c_{i}^{-}-\omega\right)\left(c_{i}^{+} \eta_{i}^{+} \exp \left(\omega \eta_{i}^{+}\right)\right. \\
& +\sum_{j=1}^{n} a_{i j}^{+} L_{j} \exp \left(\omega \tau_{i j}^{+}\right)+\sum_{j=1}^{n} b_{i j}^{+} l_{j} \exp \left(\omega \sigma_{i j}^{+}\right) \\
& \left.+\sum_{j=1}^{n} d_{i j}^{+} L_{j}^{h} \bar{\theta}_{i j} \exp (\omega)+\sum_{j=1}^{n} e_{i j}^{+} l_{j}^{k} \bar{\xi}_{i j} \exp (\omega)\right), \quad i=1,2, \ldots, n .
\end{aligned}
$$

By $\left(\mathrm{H}_{3}\right)$, for $i=1,2, \ldots, n$, we get

$$
\Theta_{i}(0)=c_{i}^{-}-\left(c_{i}^{+} \eta_{i}^{+}+\sum_{j=1}^{n} a_{i j}^{+} L_{j}+\sum_{j=1}^{n} b_{i j}^{+} l_{j}+\sum_{j=1}^{n} d_{i j}^{+} L_{j}^{h} \bar{\theta}_{i j}+\sum_{j=1}^{n} e_{i j}^{+} l_{j}^{k} \bar{\xi}_{i j}\right)>0
$$

and

$$
\Lambda_{i}(0)=c_{i}^{-}-\left(c_{i}^{+}+c_{i}^{-}\right)\left(c_{i}^{+} \eta_{i}^{+}+\sum_{j=1}^{n} a_{i j}^{+} L_{j}+\sum_{j=1}^{n} b_{i j}^{+} l_{j}+\sum_{j=1}^{n} d_{i j}^{+} L_{j}^{h} \bar{\theta}_{i j}+\sum_{j=1}^{n} e_{i j}^{+} l_{j}^{k} \bar{\xi}_{i j}\right)>0 .
$$

Since $\Theta_{i}, \Lambda_{i}$ are continuous on $[0,+\infty)$ and $\Theta_{i}(\omega), \Theta_{i}(\omega) \rightarrow-\infty$, as $\omega \rightarrow+\infty$, so there exist $\omega_{i}, \omega_{i}^{*}>0$ such that $\Theta_{i}\left(\omega_{i}\right)=\Lambda_{i}\left(\omega_{i}^{*}\right)=0$ and $\Theta_{i}(\omega)>0$ for $\omega \in\left(0, \omega_{i}\right), \Lambda_{i}(\omega)>0$ for $\omega \in\left(0, \omega_{i}^{*}\right), i=1,2, \ldots, n$.

By choosing $a=\min \left\{\omega_{1}, \omega_{2}, \ldots, \omega_{n}, \omega_{1}^{*}, \omega_{2}^{*}, \ldots, \omega_{n}^{*}\right\}$, we have $\Theta_{i}(a) \geq 0, \Lambda_{i}(a) \geq 0, i=$ $1,2, \ldots, n$. So, we can choose a positive constant $0<\lambda<\min \left\{a, \min _{1 \leq i \leq n}\left\{c_{i}^{-}\right\}\right\}$such that

$$
\Theta_{i}(\lambda)>0, \quad \Lambda_{i}(\lambda)>0, \quad i=1,2, \ldots, n,
$$

which implies that

$$
\begin{aligned}
& \frac{\exp \left(\lambda \sup _{s \in \mathbb{T}} \mu(s)\right)}{c_{i}^{-}-\lambda}\left(c_{i}^{+} \eta_{i}^{+} \exp \left(\lambda \eta_{i}^{+}\right)+\sum_{j=1}^{n} a_{i j}^{+} L_{j} \exp \left(\lambda \tau_{i j}^{+}\right)+\sum_{j=1}^{n} b_{i j}^{+} l_{j} \exp \left(\lambda \sigma_{i j}^{+}\right)\right. \\
& \left.+\sum_{j=1}^{n} d_{i j}^{+} L_{j}^{h} \bar{\theta}_{i j} \exp (\lambda)+\sum_{j=1}^{n} e_{i j}^{+} l_{j} \bar{\xi}_{i j} \exp (\lambda)\right)<1
\end{aligned}
$$


and

$$
\begin{aligned}
(1 & \left.+\frac{c_{i}^{+} \exp \left(\lambda \sup _{s \in \mathbb{T}} \mu(s)\right)}{c_{i}^{-}-\lambda}\right)\left(c_{i}^{+} \eta_{i}^{+} \exp \left(\lambda \eta_{i}^{+}\right)+\sum_{j=1}^{n} a_{i j}^{+} L_{j} \exp \left(\lambda \tau_{i j}^{+}\right)\right. \\
& \left.+\sum_{j=1}^{n} b_{i j}^{+} l_{j} \exp \left(\lambda \sigma_{i j}^{+}\right)+\sum_{j=1}^{n} d_{i j}^{+} L_{j}^{h} \bar{\theta}_{i j} \exp (\lambda)+\sum_{j=1}^{n} e_{i j}^{+} l_{j}^{k} \bar{\xi}_{i j} \exp (\lambda)\right)<1,
\end{aligned}
$$

where $i=1,2, \ldots, n$. Let

$$
M=\max _{1 \leq i \leq n}\left\{\frac{c_{i}^{-}}{c_{i}^{+} \eta_{i}^{+}+\sum_{j=1}^{n} a_{i j}^{+} L_{j}+\sum_{j=1}^{n} b_{i j}^{+} l_{j}+\sum_{j=1}^{n} d_{i j}^{+} L_{j}^{h} \bar{\theta}_{i j}+\sum_{j=1}^{n} e_{i j}^{+} l_{j}^{k} \bar{\xi}_{i j}}\right\},
$$

by $\left(\mathrm{H}_{3}\right)$ we have $M>1$. Thus

$$
\begin{aligned}
\frac{1}{M}< & \frac{\exp \left(\lambda \sup _{s \in \mathbb{T}} \mu(s)\right)}{c_{i}^{-}-\lambda}\left(c_{i}^{+} \eta_{i}^{+} \exp \left(\lambda \eta_{i}^{+}\right)+\sum_{j=1}^{n} a_{i j}^{+} L_{j} \exp \left(\lambda \tau_{i j}^{+}\right)\right. \\
& \left.+\sum_{j=1}^{n} b_{i j}^{+} l_{j} \exp \left(\lambda \sigma_{i j}^{+}\right)+\sum_{j=1}^{n} d_{i j}^{+} L_{j}^{h} \bar{\theta}_{i j} \exp (\lambda)+\sum_{j=1}^{n} e_{i j}^{+} l_{j}^{k} \bar{\xi}_{i j} \exp (\lambda)\right) .
\end{aligned}
$$

Rewrite (4.1) in the form

$$
\begin{aligned}
y_{i}^{\Delta}(t)+c_{i}(t) y_{i}(t)= & c_{i}(t) \int_{t-\eta_{i}(t)}^{t} y_{i}^{\Delta}(s) \Delta s+\sum_{j=1}^{n} a_{i j}(t) F_{j}\left(y_{j}\left(t-\tau_{i j}(t)\right)\right) \\
& +\sum_{j=1}^{n} b_{i j}(t) G_{j}\left(y_{j}^{\Delta}\left(t-\sigma_{i j}(t)\right)\right)+\sum_{j=1}^{n} d_{i j}(t) \int_{t-\delta_{i j}(t)}^{t} \theta_{i j}(s) H_{j}\left(y_{j}(s)\right) \Delta s \\
& +\sum_{j=1}^{n} e_{i j}(t) \int_{t-\zeta_{i j}(t)}^{t} \xi_{i j}(s) K_{j}\left(y_{j}^{\Delta}(s)\right) \Delta s, \quad i=1,2, \ldots, n .
\end{aligned}
$$

Multiplying the both sides of (4.4) by $e_{-c_{i}}(t, \sigma(s))$ and integrating over $\left[t_{0}, t\right]_{\mathbb{T}}$, we get

$$
\begin{aligned}
y_{i}(t)= & y_{i}\left(t_{0}\right) e_{-c_{i}}\left(t, t_{0}\right)+\int_{t_{0}}^{t} e_{-c_{i}}(t, \sigma(s))\left\{c_{i}(s) \int_{s-\eta_{i}(s)}^{s} y_{i}^{\Delta}(s) \Delta s\right. \\
& +\sum_{j=1}^{n} a_{i j}(s) F_{j}\left(y_{j}\left(s-\tau_{i j}(s)\right)\right)+\sum_{j=1}^{n} b_{i j}(s) G_{j}\left(y_{j}^{\Delta}\left(s-\sigma_{i j}(s)\right)\right) \\
& +\sum_{j=1}^{n} d_{i j}(s) \int_{s-\delta_{i j}(s)}^{s} \theta_{i j}(u) H_{j}\left(y_{j}(u)\right) \Delta u \\
& \left.+\sum_{j=1}^{n} e_{i j}(s) \int_{s-\zeta_{i j}(s)}^{s} \xi_{i j}(u) K_{j}\left(y_{j}^{\Delta}(u)\right) \Delta u\right\} \Delta s, \quad i=1,2, \ldots, n .
\end{aligned}
$$

It is easy to see that

$$
\|y(t)\|_{1}=\|\psi(t)\|_{1} \leq\|\psi\|_{\mathbb{X}} \leq M e_{\ominus \lambda}\left(t, t_{0}\right)\|\psi\|_{\mathbb{X}}, \quad \forall t \in[-\iota, 0]_{\mathbb{T}} .
$$


We claim that

$$
\|y(t)\|_{1} \leq M e_{\ominus \lambda}\left(t, t_{0}\right)\|\psi\|_{\mathbb{X}}, \quad \forall t \in(0,+\infty)_{\mathbb{T}} .
$$

To prove (4.6), we first show that for any $p>1$, the following inequality holds:

$$
\|y(t)\|_{1}<p M e_{\ominus \lambda}\left(t, t_{0}\right)\|\psi\|_{\mathbb{X}}, \quad \forall t \in(0,+\infty)_{\mathbb{T}} .
$$

If (4.7) is not true, then there must be some $t_{1} \in(0,+\infty)_{\mathbb{T}}$ and some $i_{1}, i_{2} \in\{1,2, \ldots, n\}$ such that

$$
\begin{aligned}
\left\|y\left(t_{1}\right)\right\|_{1} & =\max \left\{\left\|y\left(t_{1}\right)\right\|_{0},\left\|y^{\Delta}\left(t_{1}\right)\right\|_{0}\right\}=\max \left\{\left|y_{i_{1}}\left(t_{1}\right)\right|,\left|y_{i_{2}}^{\Delta}\left(t_{1}\right)\right|\right\} \\
& \geq p M e_{\ominus \lambda}\left(t_{1}, t_{0}\right)\|\psi\|_{\mathbb{X}}
\end{aligned}
$$

and

$$
\|y(t)\|_{1} \leq p M e_{\ominus \lambda}\left(t, t_{0}\right)\|\psi\|_{\mathbb{X}}, \quad t \in\left[-\iota, t_{1}\right]_{\mathbb{T}} .
$$

Therefore, there must exist a constant $c \geq 1$ such that

$$
\begin{aligned}
\left\|y\left(t_{1}\right)\right\|_{1} & =\max \left\{\left\|y\left(t_{1}\right)\right\|_{0},\left\|y^{\Delta}\left(t_{1}\right)\right\|_{0}\right\}=\max \left\{\left|y_{i_{1}}\left(t_{1}\right)\right|,\left|y_{i_{2}}^{\Delta}\left(t_{1}\right)\right|\right\} \\
& =c p M e_{\ominus \lambda}\left(t_{1}, t_{0}\right)\|\psi\|_{\mathbb{X}}
\end{aligned}
$$

and

$$
\|y(t)\|_{1} \leq c p M e_{\ominus \lambda}\left(t, t_{0}\right)\|\psi\|_{\mathbb{X}}, \quad t \in\left[-\iota, t_{1}\right]_{\mathbb{T}} .
$$

By (4.5), (4.8), (4.9) and $\left(\mathrm{H}_{1}\right)-\left(\mathrm{H}_{3}\right)$, we obtain

$$
\begin{aligned}
\left|y_{i_{1}}\left(t_{1}\right)\right| \leq & \|\psi\|_{X} e_{-c_{i_{1}}}\left(t_{1}, t_{0}\right)+c p M e_{\ominus \lambda}\left(t_{1}, t_{0}\right)\|\psi\|_{\mathbb{X}} \int_{t_{0}}^{t_{1}} e_{-c_{i_{1}}}\left(t_{1}, \sigma(s)\right) e_{\lambda}\left(t_{1}, \sigma(s)\right) \\
& \times\left\{c_{i_{1}}^{+} \int_{s-\eta_{i_{1}}(s)}^{s} e_{\lambda}(\sigma(s), \theta) \Delta \theta+\sum_{j=1}^{n} a_{i_{1} j}^{+} L_{j} e_{\lambda}\left(\sigma(s), s-\tau_{i_{1} j}(s)\right)\right. \\
& +\sum_{j=1}^{n} b_{i_{1} j}^{+} l_{j} e_{\lambda}\left(\sigma(s), s-\sigma_{i_{1} j}(s)\right)+\sum_{j=1}^{n} d_{i_{1} j}^{+} L_{j}^{h} \bar{\theta}_{i_{1} j} e_{\lambda}(\sigma(s), s) \\
& \left.+\sum_{j=1}^{n} e_{i_{1} j}^{+} l_{j}^{k} \bar{\xi}_{i_{j} j} e_{\lambda}(\sigma(s), s)\right\} \Delta s \\
\leq & \|\psi\|_{X} e_{-c_{i_{1}}}\left(t_{1}, t_{0}\right)+c p M e_{\ominus \lambda}\left(t_{1}, t_{0}\right)\|\psi\|_{\mathbb{X}} \int_{t_{0}}^{t_{1}} e_{-c_{i_{1}} \oplus \lambda}\left(t_{1}, \sigma(s)\right) \\
& \times\left\{c_{i_{1}}^{+} \eta_{i_{1}}^{+} e_{\lambda}\left(\sigma(s), s-\eta_{i_{1}}(s)\right)+\sum_{j=1}^{n} a_{i_{1} j}^{+} L_{j} e_{\lambda}\left(\sigma(s), s-\tau_{i_{1} j}(s)\right)\right. \\
& +\sum_{j=1}^{n} b_{i_{1} j}^{+} l_{j} e_{\lambda}\left(\sigma(s), s-\sigma_{i_{1} j}(s)\right)+\sum_{j=1}^{n} d_{i_{1} j}^{+} L_{j}^{h} \bar{\theta}_{i_{1} j} e_{\lambda}(\sigma(s), s)
\end{aligned}
$$


Li et al. Advances in Difference Equations 2014, 2014:178

Page 15 of 22

http://www.advancesindifferenceequations.com/content/2014/1/178

$$
\begin{aligned}
& \left.+\sum_{j=1}^{n} e_{i_{1} j}^{+} l_{j}^{k} \bar{\xi}_{i_{1} j} e_{\lambda}(\sigma(s), s)\right\} \Delta s \\
& \leq\|\psi\|_{X} e_{-c_{i_{1}}}\left(t_{1}, t_{0}\right)+c p M e_{\ominus \lambda}\left(t_{1}, t_{0}\right)\|\psi\|_{\mathbb{X}} \int_{t_{0}}^{t_{1}} e_{-c_{i_{1}} \oplus \lambda}\left(t_{1}, \sigma(s)\right) \\
& \times\left\{c_{i_{1}}^{+} \eta_{i_{1}}^{+} \exp \left[\lambda\left(\eta_{i_{1}}^{+}+\sup _{s \in \mathbb{T}} \mu(s)\right)\right]+\sum_{j=1}^{n} a_{i_{1} j}^{+} L_{j} \exp \left[\lambda\left(\tau_{i_{1} j}^{+}+\sup _{s \in \mathbb{T}} \mu(s)\right)\right]\right. \\
& +\sum_{j=1}^{n} b_{i_{1} j}^{+} l_{j} \exp \left[\lambda\left(\sigma_{i_{1} j}^{+}+\sup _{s \in \mathbb{T}} \mu(s)\right)\right]+\sum_{j=1}^{n} d_{i_{1} j}^{+} L_{j}^{h} \bar{\theta}_{i_{1} j} \exp \left(\lambda \sup _{s \in \mathbb{T}} \mu(s)\right) \\
& \left.+\sum_{j=1}^{n} e_{i_{1} j}^{+} l_{j}^{k} \bar{\xi}_{i_{1 j} j} \exp \left(\lambda \sup _{s \in \mathbb{T}} \mu(s)\right)\right\} \Delta s \\
& =c p M e_{\ominus \lambda}\left(t_{1}, t_{0}\right)\|\psi\|_{\mathbb{X}}\left\{\frac{1}{p M} e_{-c_{i_{1}} \oplus \lambda}\left(t_{1}, t_{0}\right)\right. \\
& +\exp \left(\lambda \sup _{s \in \mathbb{T}} \mu(s)\right)\left[c_{i_{1}}^{+} \eta_{i_{1}}^{+} \exp \left(\lambda \eta_{i_{1}}^{+}\right)+\sum_{j=1}^{n} a_{i_{1} j}^{+} L_{j} \exp \left(\lambda \tau_{i_{1} j}^{+}\right)\right. \\
& \left.+\sum_{j=1}^{n} b_{i_{1} j}^{+} l_{j} \exp \left(\lambda \sigma_{i_{1} j}^{+}\right)+\sum_{j=1}^{n} d_{i_{1} j}^{+} L_{j}^{h} \bar{\theta}_{i_{1} j}+\sum_{j=1}^{n} e_{i_{1} j}^{+} l_{j}^{k} \bar{\xi}_{i_{1} j}\right] \\
& \left.\times \int_{t_{0}}^{t_{1}} e_{-c_{i_{1}} \oplus \lambda}\left(t_{1}, \sigma(s)\right) \Delta s\right\} \\
& <c p M e_{\ominus \lambda}\left(t_{1}, t_{0}\right)\|\psi\|_{\mathbb{X}}\left\{\frac{1}{M} e_{-\left(c_{i_{1}}^{-}-\lambda\right)}\left(t_{1}, t_{0}\right)+\exp \left(\lambda \sup _{s \in \mathbb{T}} \mu(s)\right)\left[c_{i_{1}}^{+} \eta_{i_{1}}^{+} \exp \left(\lambda \eta_{i_{1}}^{+}\right)\right.\right. \\
& \left.+\sum_{j=1}^{n} a_{i_{1} j}^{+} L_{j} \exp \left(\lambda \tau_{i_{1} j}^{+}\right)+\sum_{j=1}^{n} b_{i_{1} j}^{+} l_{j} \exp \left(\lambda \sigma_{i_{1} j}^{+}\right)+\sum_{j=1}^{n} d_{i_{1} j}^{+} L_{j}^{h} \bar{\theta}_{i_{1} j}+\sum_{j=1}^{n} e_{i_{1} j}^{+} l_{j}^{k} \bar{\xi}_{i_{1} j}\right] \\
& \left.\times \frac{1}{-\left(c_{i_{1}}^{-}-\lambda\right)} \int_{t_{0}}^{t_{1}}-\left(c_{i_{1}}^{-}-\lambda\right) e_{-\left(c_{i_{1}}^{-}-\lambda\right)}\left(t_{1}, \sigma(s)\right) \Delta s\right\} \\
& \leq c p M e_{\ominus \lambda}\left(t_{1}, t_{0}\right)\|\psi\|_{\mathbb{X}}\left\{\left[\frac{1}{M}-\frac{\exp \left(\lambda \sup _{s \in \mathbb{T}} \mu(s)\right)}{c_{i_{1}}^{-}-\lambda}\left(c_{i_{1}}^{+} \eta_{i_{1}}^{+} \exp \left(\lambda \eta_{i_{1}}^{+}\right)\right.\right.\right. \\
& +\sum_{j=1}^{n} a_{i_{1} j}^{+} L_{j} \exp \left(\lambda \tau_{i_{1} j}^{+}\right)+\sum_{j=1}^{n} b_{i_{1} j}^{+} l_{j} \exp \left(\lambda \sigma_{i_{1} j}^{+}\right)+\sum_{j=1}^{n} d_{i_{1} j}^{+} L_{j}^{h} \bar{\theta}_{i_{1} j} \\
& \left.\left.+\sum_{j=1}^{n} e_{i_{1}}^{+} l_{j}^{k} \bar{\xi}_{i_{1} j}\right)\right] e_{-\left(c_{i_{1}}^{-}-\lambda\right)}\left(t_{1}, t_{0}\right)+\frac{\exp \left(\lambda \sup _{s \in \mathbb{T}} \mu(s)\right)}{c_{i_{1}}^{-}-\lambda}\left(c_{i_{1}}^{+} \eta_{i_{1}}^{+} \exp \left(\lambda \eta_{i_{1}}^{+}\right)\right. \\
& +\sum_{j=1}^{n} a_{i_{1} j}^{+} L_{j} \exp \left(\lambda \tau_{i_{1} j}^{+}\right)+\sum_{j=1}^{n} b_{i_{1}}^{+} l_{j} \exp \left(\lambda \sigma_{i_{1} j}^{+}\right) \\
& \left.\left.+\sum_{j=1}^{n} d_{i_{1} j}^{+} L_{j}^{h} \bar{\theta}_{i_{1} j} \exp (\lambda)+\sum_{j=1}^{n} e_{i_{1} j}^{+} l_{j}^{k} \bar{\xi}_{i_{1} j} \exp (\lambda)\right)\right\} \\
& <c p M e_{\ominus \lambda}\left(t_{1}, t_{0}\right)\|\psi\|_{\mathbb{X}} .
\end{aligned}
$$


By Lemma 2.4 and (4.5), we have, for $i=1,2, \ldots, n$,

$$
\begin{aligned}
y_{i}^{\Delta}(t)= & -c_{i}(t) y_{i}\left(t_{0}\right) e_{-c_{i}}\left(t, t_{0}\right)+\left(c_{i}(t) \int_{t-\eta_{i}(t)}^{t} y_{i}^{\Delta}(s) \Delta s+\sum_{j=1}^{n} a_{i j}(t) F_{j}\left(y_{j}\left(t-\tau_{i j}(t)\right)\right)\right. \\
& +\sum_{j=1}^{n} b_{i j}(t) G_{j}\left(y_{j}^{\Delta}\left(t-\sigma_{i j}(t)\right)\right)+\sum_{j=1}^{n} d_{i j}(t) \int_{t-\delta_{i j}(t)}^{t} \theta_{i j}(s) H_{j}\left(y_{j}(s)\right) \Delta s \\
& +\sum_{j=1}^{n} e_{i j}(t) \int_{t-\zeta_{i j}(t)}^{t} \xi_{i j}(s) K_{j}\left(y_{j}^{\Delta}(s)\right) \Delta s \\
& +\int_{t_{0}}^{t}-c_{i}(t) e_{-c_{i}}(t, \sigma(s))\left\{c_{i}(s) \int_{s-\eta_{i}(s)}^{s} y_{i}^{\Delta}(u) \Delta u+\sum_{j=1}^{n} a_{i j}(s) F_{j}\left(y_{j}\left(s-\tau_{i j}(s)\right)\right)\right. \\
& +\sum_{j=1}^{n} b_{i j}(s) G_{j}\left(y_{j}^{\Delta}\left(s-\sigma_{i j}(s)\right)\right)+\sum_{j=1}^{n} d_{i j}(s) \int_{s-\delta_{i j}(s)}^{s} \theta_{i j}(u) H_{j}\left(y_{j}(u)\right) \Delta u \\
& \left.+\sum_{j=1}^{n} e_{i j}(s) \int_{s-\zeta_{i j}(s)}^{s} \xi_{i j}(u) K_{j}\left(y_{j}^{\Delta}(u)\right) \Delta u\right\} \Delta s .
\end{aligned}
$$

Thus, it follows from (4.8), (4.9) and (4.11) that

$$
\begin{aligned}
& \left|y_{i_{2}}^{\Delta}\left(t_{1}\right)\right| \leq c_{i_{2}}^{+} e_{-c_{i_{2}}}\left(t_{1}, t_{0}\right)\|\psi\|_{\mathbb{X}}+c p M e_{\ominus \lambda}\left(t_{1}, t_{0}\right)\|\psi\|_{X}\left(c_{i_{2}}^{+} \int_{t_{1}-\eta_{i_{2}}\left(t_{1}\right)}^{t_{1}} e_{\lambda}\left(t_{1}, \theta\right) \Delta \theta\right. \\
& +\sum_{j=1}^{n} a_{i_{2} j}^{+} L_{j} e_{\lambda}\left(t_{1}, t_{1}-\tau_{i_{2} j}\left(t_{1}\right)\right)+\sum_{j=1}^{n} b_{i_{2} j}^{+} l_{j} e_{\lambda}\left(t_{1}, t_{1}-\sigma_{i_{2} j}\left(t_{1}\right)\right) \\
& \left.+\sum_{j=1}^{n} d_{i_{2} j}^{+} L_{j}^{h} \bar{\theta}_{i_{2} j} e_{\lambda}\left(t_{1}, t_{1}\right)+\sum_{j=1}^{n} e_{i_{2} j}^{+} l_{j}^{k} \bar{\xi}_{i_{2} j} e_{\lambda}\left(t_{1}, t_{1}\right)\right) \\
& +c_{i_{2}}^{+} c p M e_{\ominus \lambda}\left(t_{1}, t_{0}\right)\|\psi\|_{\mathbb{X}} \int_{t_{0}}^{t_{1}} e_{-c_{i_{2}}}\left(t_{1}, \sigma(s)\right) e_{\lambda}\left(t_{1}, \sigma(s)\right) \\
& \times\left\{c_{i_{2}}^{+} \int_{s-\eta_{i_{2}}(s)}^{s} e_{\lambda}(\sigma(s), \theta) \Delta \theta\right. \\
& +\sum_{j=1}^{n} a_{i_{2} j}^{+} L_{j} e_{\lambda}\left(\sigma(s), s-\tau_{i_{2} j}(s)\right)+\sum_{j=1}^{n} b_{i_{2 j}}^{+} l_{j} e_{\lambda}\left(\sigma(s), s-\sigma_{i_{2} j}(s)\right) \\
& \left.+\sum_{j=1}^{n} d_{i_{2} j}^{+} L_{j}^{h} \bar{\theta}_{i_{2} j} e_{\lambda}(\sigma(s), s)+\sum_{j=1}^{n} e_{i_{2 j}}^{+} l_{j}^{k} \bar{\xi}_{i_{2} j} e_{\lambda}(\sigma(s), s)\right\} \Delta s \\
& \leq c_{i_{2}}^{+} e_{-c_{i_{2}}}\left(t_{1}, t_{0}\right)\|\psi\|_{\mathbb{X}}+c p M e_{\ominus \lambda}\left(t_{1}, t_{0}\right)\|\psi\|_{\mathbb{X}}\left(c_{i_{2}}^{+} \eta_{i_{2}}^{+} e_{\lambda}\left(t_{1}, t_{1}-\eta_{i_{2}}\left(t_{1}\right)\right)\right. \\
& +\sum_{j=1}^{n} a_{i_{2} j}^{+} L_{j} e_{\lambda}\left(t_{1}, t_{1}-\tau_{i_{2} j}\left(t_{1}\right)\right)+\sum_{j=1}^{n} b_{i_{2 j}}^{+} l_{j} e_{\lambda}\left(t_{1}, t_{1}-\sigma_{i_{2} j}\left(t_{1}\right)\right) \\
& \left.+\sum_{j=1}^{n} d_{i_{2} j}^{+} L_{j}^{h} \bar{\theta}_{i_{2} j}+\sum_{j=1}^{n} e_{i_{2} j}^{+} l_{j}^{k} \bar{\xi}_{i_{2} j}\right)+c_{i_{2}}^{+} c p M e_{\ominus \lambda}\left(t_{1}, t_{0}\right)\|\psi\|_{\mathbb{X}} \int_{t_{0}}^{t_{1}} e_{-c_{i_{2}} \oplus \lambda}\left(t_{1}, \sigma(s)\right)
\end{aligned}
$$


Li et al. Advances in Difference Equations 2014, 2014:178

Page 17 of 22

http://www.advancesindifferenceequations.com/content/2014/1/178

$$
\begin{aligned}
& \times\left\{c_{i_{2}}^{+} \eta_{i_{2}}^{+} e_{\lambda}\left(\sigma(s), s-\eta_{i_{2}}(s)\right)+\sum_{j=1}^{n} a_{i_{2} j}^{+} L_{j} e_{\lambda}\left(\sigma(s), s-\tau_{i_{2} j}(s)\right)\right. \\
& +\sum_{j=1}^{n} b_{i_{2} j}^{+} l_{j} e_{\lambda}\left(\sigma(s), s-\sigma_{i_{2} j}(s)\right)+\sum_{j=1}^{n} d_{i_{2} j}^{+} L_{j}^{h} \bar{\theta}_{i_{2} j} e_{\lambda}(\sigma(s), s) \\
& \left.+\sum_{j=1}^{n} e_{i_{2} j}^{+} l_{j}^{k} \bar{\xi}_{i_{2} j} e_{\lambda}(\sigma(s), s)\right\} \Delta s \\
& \leq c_{i_{2}}^{+} e_{-c_{i_{2}}}\left(t_{1}, t_{0}\right)\|\psi\|_{\mathbb{X}}+c p M e_{\ominus \lambda}\left(t_{1}, t_{0}\right)\|\psi\|_{\mathbb{X}}\left(c_{i_{2}}^{+} \eta_{i_{2}}^{+} \exp \left(\lambda \eta_{i_{2}}^{+}\right)\right. \\
& +\sum_{j=1}^{n} a_{i_{2} j}^{+} L_{j} \exp \left(\lambda \tau_{i_{2} j}^{+}\right)+\sum_{j=1}^{n} b_{i_{2} j}^{+} l_{j} \exp \left(\lambda \sigma_{i_{2} j}^{+}\right) \\
& \left.+\sum_{j=1}^{n} d_{i_{2} j}^{+} L_{j}^{h} \bar{\theta}_{i_{2} j} \exp (\lambda)+\sum_{j=1}^{n} e_{i_{2} j}^{+} j_{j}^{k} \bar{\xi}_{i_{2} j} \exp (\lambda)\right) \\
& \times\left(1+c_{i_{2}}^{+} \exp \left(\lambda \sup _{s \in \mathbb{T}} \mu(s)\right) \int_{t_{0}}^{t_{1}} e_{-c_{i_{2}} \oplus \lambda}\left(t_{1}, \sigma(s)\right) \Delta s\right) \\
& <c p M e_{\ominus \lambda}\left(t_{1}, t_{0}\right)\|\psi\|_{\mathbb{X}}\left\{\frac{c_{i_{2}}^{+}}{M} e_{-c_{i_{2}} \oplus \lambda}\left(t_{1}, t_{0}\right)+\left(c_{i_{2}}^{+} \eta_{i_{2}}^{+} \exp \left(\lambda \eta_{i_{2}}^{+}\right)\right.\right. \\
& +\sum_{j=1}^{n} a_{i_{2} j}^{+} L_{j} \exp \left(\lambda \tau_{i_{2 j}}^{+}\right)+\sum_{j=1}^{n} b_{i_{2 j}}^{+} l_{j} \exp \left(\lambda \sigma_{i_{2} j}^{+}\right) \\
& \left.+\sum_{j=1}^{n} d_{i_{2} j}^{+} L_{j}^{h} \bar{\theta}_{i_{2} j} \exp (\lambda)+\sum_{j=1}^{n} e_{i_{2} j}^{+} j_{j}^{k} \bar{\xi}_{i_{2 j} j} \exp (\lambda)\right) \\
& \left.\times\left(1+c_{i_{2}}^{+} \exp \left(\lambda \sup _{s \in \mathbb{T}} \mu(s)\right) \int_{t_{0}}^{t_{1}} e_{-c_{i_{2}} \oplus \lambda}\left(t_{1}, \sigma(s)\right) \Delta s\right)\right\} \\
& \leq c p M e_{\ominus \lambda}\left(t_{1}, t_{0}\right)\|\psi\|_{\mathbb{X}}\left\{\frac{c_{i_{2}}^{+}}{M} e_{-\left(c_{i_{2}}^{-}-\lambda\right)}\left(t_{1}, t_{0}\right)+\left(c_{i_{2}}^{+} \eta_{i_{2}}^{+} \exp \left(\lambda \eta_{i_{2}}^{+}\right)\right.\right. \\
& +\sum_{j=1}^{n} a_{i_{2} j}^{+} L_{j} \exp \left(\lambda \tau_{i_{2 j}}^{+}\right)+\sum_{j=1}^{n} b_{i_{2} j}^{+} l_{j} \exp \left(\lambda \sigma_{i_{2} j}^{+}\right) \\
& \left.+\sum_{j=1}^{n} d_{i_{2} j}^{+} L_{j}^{h} \bar{\theta}_{i_{2} j} \exp (\lambda)+\sum_{j=1}^{n} e_{i_{2} j}^{+} j_{j}^{k} \bar{\xi}_{i_{2} j} \exp (\lambda)\right) \\
& \left.\times\left(1+c_{i_{2}}^{+} \exp \left(\lambda \sup _{s \in \mathbb{T}} \mu(s)\right) \frac{1}{-\left(c_{i_{2}}^{-}-\lambda\right)}\left(e_{-\left(c_{i_{2}}^{-}-\lambda\right)}\left(t_{1}, t_{0}\right)-1\right)\right)\right\} \\
& \leq c p M e_{\ominus \lambda}\left(t_{1}, t_{0}\right)\|\psi\|_{\mathbb{X}}\left\{\left[\frac{1}{M}-\frac{\exp \left(\lambda \sup _{s \in \mathbb{T}} \mu(s)\right)}{c_{i_{2}}^{-}-\lambda}\left(c_{i_{2}}^{+} \eta_{i_{2}}^{+} \exp \left(\lambda \eta_{i_{2}}^{+}\right)\right.\right.\right. \\
& +\sum_{j=1}^{n} a_{i_{2} j}^{+} L_{j} \exp \left(\lambda \tau_{i_{2} j}^{+}\right)+\sum_{j=1}^{n} b_{i_{2} j}^{+} l_{j} \exp \left(\lambda \sigma_{i_{2} j}^{+}\right) \\
& \left.\left.+\sum_{j=1}^{n} d_{i_{2} j}^{+} L_{j}^{h} \bar{\theta}_{i_{2} j} \exp (\lambda)+\sum_{j=1}^{n} e_{i_{2} j}^{+} l_{j}^{k} \bar{\xi}_{i_{2 j}} \exp (\lambda)\right)\right] c_{i_{2}}^{+} e_{-\left(c_{i_{2}}^{-}-\lambda\right)}\left(t_{1}, t_{0}\right)
\end{aligned}
$$




$$
\begin{aligned}
& +\left(1+\frac{c_{i_{2}}^{+} \exp \left(\lambda \sup _{s \in \mathbb{T}} \mu(s)\right)}{c_{i_{2}}^{-}-\lambda}\right)\left(c_{i_{2}}^{+} \eta_{i_{2}}^{+} \exp \left(\lambda \eta_{i_{2}}^{+}\right)+\sum_{j=1}^{n} a_{i_{2} j}^{+} L_{j} \exp \left(\lambda \tau_{i_{2} j}^{+}\right)\right. \\
& \left.\left.+\sum_{j=1}^{n} b_{i_{2} j}^{+} l_{j} \exp \left(\lambda \sigma_{i_{2} j}^{+}\right)+\sum_{j=1}^{n} d_{i_{2} j}^{+} L_{j}^{h} \bar{\theta}_{i_{2} j} \exp (\lambda)+\sum_{j=1}^{n} e_{i_{2 j}}^{+} j_{j}^{k} \bar{\xi}_{i_{2} j} \exp (\lambda)\right)\right\} \\
& <c p M e_{\ominus \lambda}\left(t_{1}, t_{0}\right)\|\psi\|_{\mathbb{X} .}
\end{aligned}
$$

In view of (4.10) and (4.12), we get

$$
\left\|y\left(t_{1}\right)\right\|_{1}<c p M e_{\ominus \lambda}\left(t_{1}, t_{0}\right)\|\psi\|_{\mathbb{X}}
$$

which contradicts (4.8), and so (4.7) holds. Letting $p \rightarrow 1$, then (4.6) holds. Hence, the almost periodic solution of system (1.1) is globally exponentially stable. This completes the proof.

Remark 4.1 When $\mathbb{T}=\mathbb{R}, \eta_{i}(t)=d_{i j}(t)=e_{i j}(t) \equiv 0, i=1,2, \ldots, n$, Theorem 3.1 and Theorem 4.1 are reduced to Theorem 2.3 and Theorem 3.1 in [37], respectively.

Remark 4.2 According to Theorem 3.1 and Theorem 4.1, we see that the existence and exponential stability of almost periodic solutions for system (1.1) only depend on time delays $\eta_{i}$ (the delays in the leakage term) and do not depend on time delays $\tau_{i j}$ and $\sigma_{i j}$.

\section{An example}

In this section, we give an example to illustrate the feasibility and effectiveness of our results obtained in Sections 3 and 4.

Example 5.1 Let $n=3$. Consider the following neutral Hopfield neural network on time scale $\mathbb{T}$ :

$$
\begin{aligned}
x_{i}^{\Delta}(t)= & -c_{i}(t) x_{i}\left(t-\eta_{i}(t)\right)+\sum_{j=1}^{3} a_{i j}(t) f_{j}\left(x_{j}\left(t-\tau_{i j}(t)\right)\right) \\
& +\sum_{j=1}^{3} d_{i j}(t) \int_{t-\delta_{i j}(t)}^{t} \theta_{i j}(s) h_{j}\left(x_{j}(s)\right) \Delta s+\sum_{j=1}^{3} b_{i j}(t) g_{j}\left(x_{j}^{\Delta}\left(t-\sigma_{i j}(t)\right)\right) \\
& +\sum_{j=1}^{3} e_{i j}(t) \int_{t-\zeta_{i j}(t)}^{t} \xi_{i j}(s) k_{j}\left(x_{j}^{\Delta}(s)\right) \Delta s+I_{i}(t)
\end{aligned}
$$

where $i=1,2,3$ and the coefficients are as follows:

$$
\begin{aligned}
& c_{1}(t)=0.5+0.1|\sin t|, \quad c_{2}(t)=0.6+0.3|\cos \sqrt{2} t|, \quad c_{3}(t)=0.8+0.1|\sin 2 t|, \\
& \eta_{1}(t)=\frac{1+|\sin \sqrt{2} t|}{200}, \quad \eta_{2}(t)=\frac{1.8+0.2|\cos \sqrt{2} t|}{100}, \quad \eta_{3}(t)=\frac{2.5+0.5|\sin 2 t|}{200}, \\
& \left(a_{i j}(t)\right)_{3 \times 3}=\left(\begin{array}{ccc}
0.08|\sin t| & 0.15|\cos \sqrt{2} t| & 0.06|\cos t| \\
0.15|\cos t| & 0.12|\cos \sqrt{2} t| & 0.04|\sin \sqrt{2} t| \\
0.10|\sin t| & 0.08|\sin \sqrt{2} t| & 0.09|\sin t|
\end{array}\right),
\end{aligned}
$$




$$
\begin{aligned}
& \left(b_{i j}(t)\right)_{3 \times 3}=\left(\begin{array}{ccc}
0.10|\sin t| & 0.06|\sin \sqrt{2} t| & 0.05|\cos t| \\
0.06|\sin t| & 0.03|\cos \sqrt{2} t| & 0.08|\sin \sqrt{2} t| \\
0.12|\cos t| & 0.04|\sin \sqrt{2} t| & 0.07|\sin t|
\end{array}\right), \\
& \left(d_{i j}(t)\right)_{3 \times 3}=\left(\begin{array}{ccc}
0.02|\cos t| & 0.15|\sin \sqrt{2} t| & 0.01|\cos t| \\
0.05|\sin t| & 0.12|\cos \sqrt{2} t| & 0.03|\sin \sqrt{2} t| \\
0.03|\sin t| & 0.08|\cos \sqrt{2} t| & 0.01|\sin t|
\end{array}\right), \\
& \left(e_{i j}(t)\right)_{3 \times 3}=\left(\begin{array}{ccc}
0.02|\cos t| & 0.01|\sin \sqrt{2} t| & 0.05|\cos t| \\
0.01|\sin t| & 0.03|\cos \sqrt{2} t| & 0.04|\cos \sqrt{2} t| \\
0.02|\cos t| & 0.04|\sin \sqrt{2} t| & 0.01|\sin t|
\end{array}\right), \\
& f_{1}(x)=0.2|x|, \quad f_{2}(x)=0.4|\sin x|, \quad f_{3}(x)=|x|, \\
& g_{1}(x)=0.3|\cos x|, \quad g_{2}(x)=0.1|x|, \quad g_{3}(x)=0.5|\sin x|, \\
& h_{1}(x)=0.1|x|, \quad h_{2}(x)=0.4|\sin x|, \quad h_{3}(x)=0.2|x|, \\
& k_{1}(x)=0.3|\cos x|, \quad k_{2}(x)=k_{3}(x)=0.2|x|, \\
& \theta_{i j}(u)=\exp (-2 u), \quad \xi_{i j}(u)=\exp (-4 u), \\
& \delta_{i j}(t)=0.001|\sin t|, \quad \zeta_{i j}(t)=0.002|\cos t|, \quad i, j=1,2,3 .
\end{aligned}
$$

Take $\tau_{i j}>0, \sigma_{i j}>0, I_{i}(t)(i, j=1,2,3)$ to be arbitrary almost periodic functions. If $\mathbb{T}=\mathbb{R}$, then $\mu(t)=0$ and if $\mathbb{T}=\mathbb{Z}$, then $\mu(t)=1$. By calculating, we can easily check, for above two cases, that $-c_{i} \in \mathcal{R}^{+}, r \approx 0.7961<1$. By Theorem 3.1 and Theorem 4.1, we know that system (5.1) has a unique almost periodic solution that is globally exponentially stable. This shows that the almost periodicity of system (5.1) does not depend on time scale $\mathbb{T}$. In particular, the continuous-time neural network and the discrete-time analogue described by (5.1) have the same dynamical behaviors (see Figures 1-4).

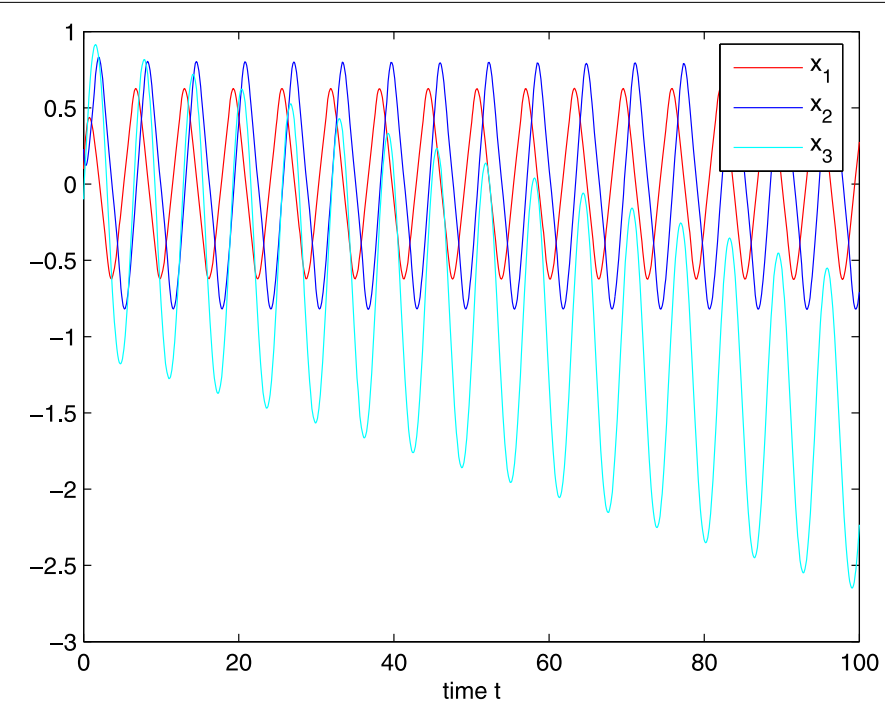

Figure 1 Continuous situation $x_{1}, x_{2}, x_{3}$ with time $t$. 


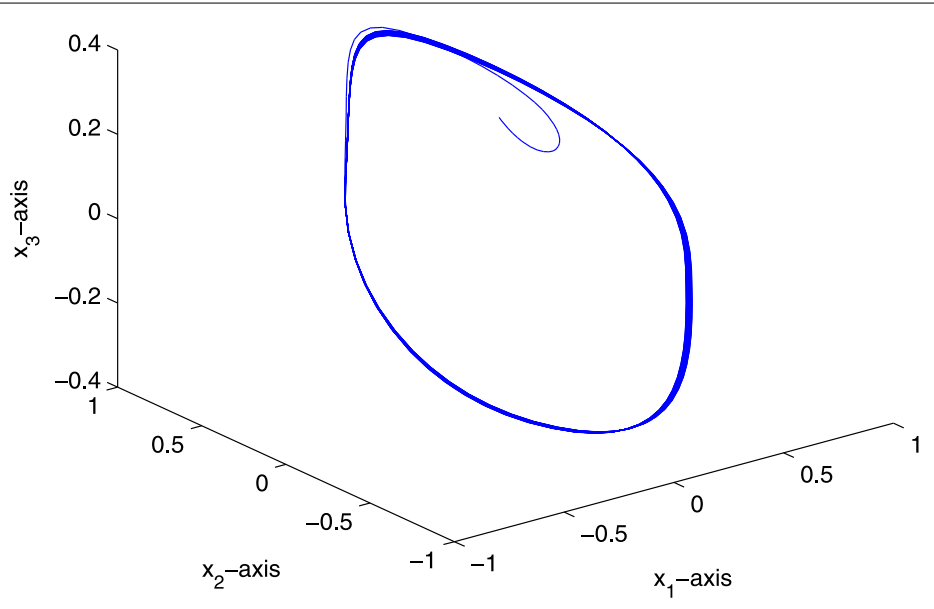

Figure 2 Continuous situation $x_{1}, x_{2}, x_{3}$.

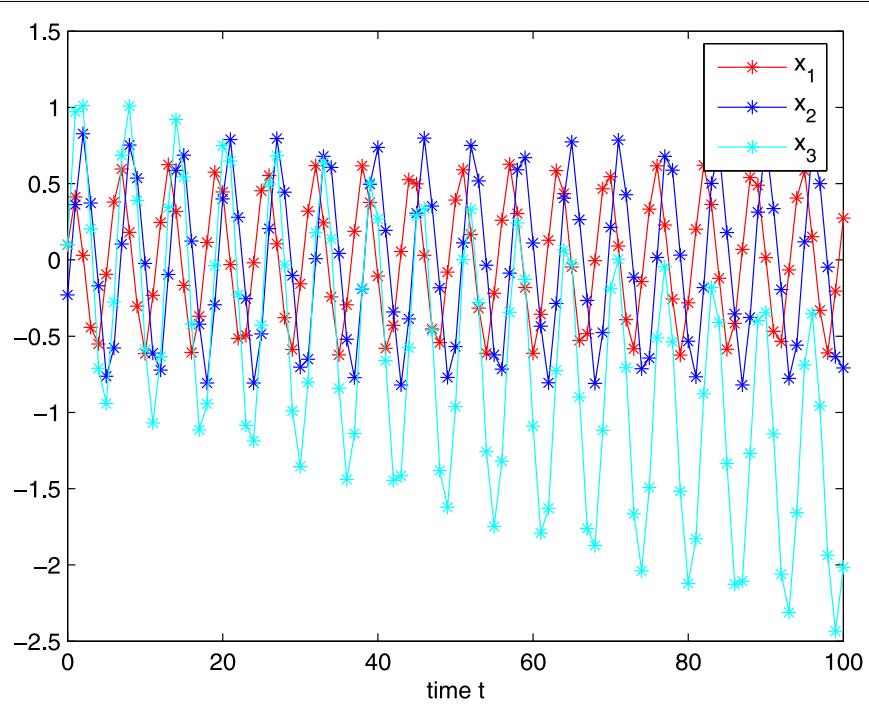

Figure 3 Discrete situation $x_{1}, x_{2}, x_{3}$ with time $t$.

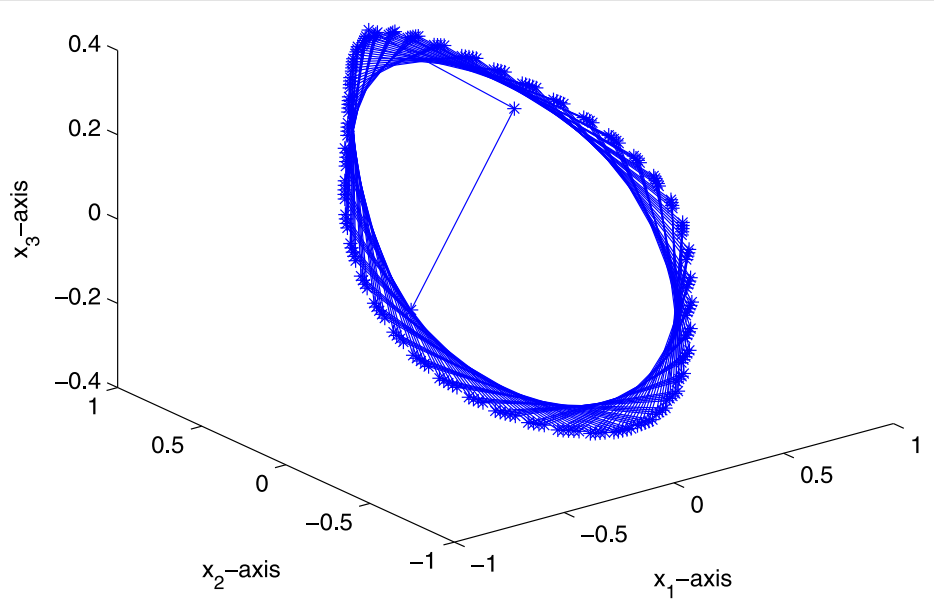

Figure 4 Discrete situation $x_{1}, x_{2}, x_{3}$. 


\section{Conclusion}

In this paper, a class of neutral delay Hopfield neural networks with neutral time-varying delays in the leakage term on time scales is investigated. For the model, we have given some sufficient conditions ensuring the existence and global exponential stability of almost periodic solutions by using the exponential dichotomy of linear dynamic equations on time scales, Banach's fixed point theorem and the theory of calculus on time scales. These obtained results are new and complement previously known results. Furthermore, a simple example is given to demonstrate the effectiveness of our results and the example also shows that the continuous-time neural network and the discrete-time analogue have the same dynamical behaviors.

\section{Competing interests}

The authors declare that they have no competing interests.

\section{Authors' contributions}

All authors contributed equally to the manuscript and typed, read and approved the final manuscript.

\section{Acknowledgements}

This study was supported by the National Natural Sciences Foundation of People's Republic of China under Grant 11361072.

Received: 15 March 2014 Accepted: 30 June 2014 Published: 22 July 2014

\section{References}

1. Gu, HB, Jiang, HJ, Teng, ZD: Existence and globally exponential stability of periodic solution of BAM neural networks with impulses and recent-history distributed delays. Neurocomputing 71, 813-822 (2008)

2. Huang, CX, Cao, JD: Almost sure exponential stability of stochastic cellular neural networks with unbounded distributed delays. Neurocomputing 72, 3352-3356 (2009)

3. Kwon, OM, Park, JH: Delay-dependent stability for uncertain cellular neural networks with discrete and distribute time-varying delays. J. Franklin Inst. 345, 766-778 (2008)

4. Li, YK, Yang, L: Anti-periodic solutions for Cohen-Grossberg neural networks with bounded and unbounded delays. Commun. Nonlinear Sci. Numer. Simul. 14, 3134-3140 (2009)

5. Li, YK: Global exponential stability of BAM neural networks with delays and impulses. Chaos Solitons Fractals 24 279-285 (2005)

6. Li, CZ, Li, YK, Ye, Y: Exponential stability of fuzzy Cohen-Grossberg neural networks with time delays and impulsive effects. Commun. Nonlinear Sci. Numer. Simul. 15, 3599-3606 (2010)

7. Cai, Z, Huang, L, Guo, Z, Chen, X: On the periodic dynamics of a class of time-varying delayed neural networks via differential inclusions. Neural Netw. 33, 97-113 (2012)

8. Akhmet, MU, Yılmaz, E: Global exponential stability of neural networks with non-smooth and impact activations. Neural Netw. 34, 18-27 (2012)

9. Kwon, OM, Park, JH, Lee, SM, Cha, EJ: New results on exponential passivity of neural networks with time-varying delays. Nonlinear Anal., Real World Appl. 13, 1593-1599 (2012)

10. Liu, PC, Yi, FQ, Guo, Q, Yang, J, Wu, W: Analysis on global exponential robust stability of reaction-diffusion neural networks with S-type distributed delays. Physica D 237, 475-485 (2008)

11. Mathiyalagan, K, Sakthivel, R, Marshal Anthoni, S: Exponential stability result for discrete-time stochastic fuzzy uncertain neural networks. Phys. Lett. A 376, 901-912 (2012)

12. Mohamad, S, Gopalsamy, K, Akca, H: Exponential stability of artificial neural networks with distributed delays and large impulses. Nonlinear Anal., Real World Appl. 9, 872-888 (2008)

13. Sakthivel, R, Mathiyalagan, K, Marshal Anthoni, S: Design of a passification controller for uncertain fuzzy Hopfield neural networks with time-varying delays. Phys. Scr. 84, 045024 (2011)

14. Zhou, DM, Zhang, LM, Cao, JD: On global exponential stability of cellular neural networks with Lipschitz-continuous activation function and variable delays. Appl. Math. Comput. 151(2), 379-392 (2004)

15. Li, YK, Fan, XL: Existence and globally exponential stability of almost periodic solution for Cohen-Grossberg BAM neural networks with variable coefficients. Appl. Math. Model. 33, 2114-2120 (2009)

16. Li, YK, Liu, CC, Zhu, LF: Global exponential stability of periodic solution for shunting inhibitory CNNs with delays. Phys. Lett. A 337, 46-54 (2005)

17. Li, YK: Global stability and existence of periodic solutions of discrete delayed cellular neural networks. Phys. Lett. A 333, 51-61 (2004)

18. Li, YK, Zhang, TW, Xing, ZW: The existence of nonzero almost periodic solution for Cohen-Grossberg neural networks with continuously distributed delays and impulses. Neurocomputing 73, 3105-3113 (2010)

19. Li, YK, Zhao, KH: Robust stability of delayed reaction-diffusion recurrent neural networks with Dirichlet boundary conditions on time scales. Neurocomputing 74, 1632-1637 (2011)

20. Song, QK, Cao, JD: Stability analysis of Cohen-Grossberg neural network with both time-varying and continuously distributed delays. J. Comput. Appl. Math. 197, 188-203 (2006)

21. Liu, BW: Global exponential stability for BAM neural networks with time-varying delays in the leakage terms. Nonlinear Anal., Real World Appl. 14, 559-566 (2013) 
22. Balasubramaniam, P, Kalpana, M, Rakkiyappan, R: Existence and global asymptotic stability of fuzzy cellular neural networks with time delay in the leakage term and unbounded distributed delays. Circuits Syst. Signal Process. 30 1595-1616 (2011)

23. Li, X, Cao, J: Delay-dependent stability of neural networks of neutral type with time delay in the leakage term. Nonlinearity 23, 1709-1726 (2010)

24. Li, X, Rakkiyappan, R, Balasubramanian, P: Existence and global stability analysis of equilibrium of fuzzy cellular neural networks with time delay in the leakage term under impulsive perturbations. J. Franklin Inst. 348, 135-155 (2011)

25. Balasubramanian, P, Nagamani, G, Rakkiyappan, R: Passivity analysis for neural networks of neutral type with Markovian jumping parameters and time delay in the leakage term. Commun. Nonlinear Sci. Numer. Simul. 16 4422-4437 (2011)

26. Lakshmanan, S, Park, JH, Jung, HY, Balasubramaniam, P: Design of state estimator for neural networks with leakage, discrete and distributed delays. Appl. Math. Comput. 218, 11297-11310 (2012)

27. Balasubramaniam, P, Vembarasan, V, Rakkiyappan, R: Leakage delays in T-S fuzzy cellular neural networks. Neura Process. Lett. 33, 111-136 (2011)

28. Li, X, Fu, X, Balasubramaniam, P, Rakkiyappan, R: Existence, uniqueness and stability analysis of recurrent neural networks with time delay in the leakage term under impulsive perturbations. Nonlinear Anal., Real World Appl. 11, 4092-4108 (2010)

29. Gopalsamy, K: Leakage delays in BAM. J. Math. Anal. Appl. 325, 1117-1132 (2007)

30. Li, C, Huang, T: On the stability of nonlinear systems with leakage delay. J. Franklin Inst. 346, 366-377 (2009)

31. Peng, S: Global attractive periodic solutions of BAM neural networks with continuously distributed delays in the leakage terms. Nonlinear Anal., Real World Appl. 11, 2141-2151 (2010)

32. Balasubramaniam, P, Kalpana, M, Rakkiyappan, R: State estimation for fuzzy cellular neural networks with time delay in the leakage term, discrete and unbounded distributed delays. Comput. Math. Appl. 62, 3959-3972 (2011)

33. Li, YK, Li, YQ: Existence and exponential stability of almost periodic solution for neutral delay BAM neural networks with time-varying delays in leakage terms. J. Franklin Inst. 350, 2808-2825 (2013)

34. Chen, ZB: A shunting inhibitory cellular neural network with leakage delays and continuously distributed delays of neutral type. Neural Comput. Appl. 23, 2429-2434 (2013)

35. Zhao, CH, Wang, ZY: Exponential convergence of a SICNN with leakage delays and continuously distributed delays of neutral type. Neural Process. Lett. (2014). doi:10.1007/s11063-014-9341-1

36. Li, YK, Zhao, L, Chen, XR: Existence of periodic solutions for neutral type cellular neural networks with delays. Appl. Math. Model. 36, 1173-1183 (2012)

37. Bai, C: Global stability of almost periodic solutions of Hopfield neural networks with neutral time-varying delays. Appl. Math. Comput. 203, 72-79 (2008)

38. Xiao, B: Existence and uniqueness of almost periodic solutions for a class of Hopfield neural networks with neutral delays. Appl. Math. Lett. 22, 528-533 (2009)

39. Park, JH, Park, CH, Kwon, OM, Lee, SM: A new stability criterion for bidirectional associative memory neural networks of neutral-type. Appl. Math. Comput. 199, 716-722 (2008)

40. Rakkiyappan, R, Balasubramaniam, P: New global exponential stability results for neutral type neural networks with distributed time delays. Neurocomputing 71, 1039-1045 (2008)

41. Rakkiyappan, R, Balasubramaniam, P: LMI conditions for global asymptotic stability results for neutral-type neural networks with distributed time delays. Appl. Math. Comput. 204, 317-324 (2008)

42. Zhang, Z, Liu, W, Zhou, D: Global asymptotic stability to a generalized Cohen-Grossberg BAM neural networks of neutral type delays. Neural Netw. 25, 94-105 (2012)

43. Liu, PL: Improved delay-dependent stability of neutral type neural networks with distributed delays. ISA Trans. $\mathbf{5 2}$ 717-724 (2013)

44. Li, YK, Chen, XR, Zhao, L: Stability and existence of periodic solutions to delayed Cohen-Grossberg BAM neural networks with impulses on time scales. Neurocomputing 72, 1621-1630 (2009)

45. Li, YK, Shu, JY: Anti-periodic solutions to impulsive shunting inhibitory cellular neural networks with distributed delays on time scales. Commun. Nonlinear Sci. Numer. Simul. 16, 3326-3336 (2011)

46. Li, YK, Wang, C: Almost periodic solutions of shunting inhibitory cellular neural networks on time scales. Commun. Nonlinear Sci. Numer. Simul. 17, 3258-3266 (2012)

47. Liang, T, Yang, YQ, Liu, Y, Li, L: Existence and global exponential stability of almost periodic solutions to Cohen-Grossberg neural networks with distributed delays on time scales. Neurocomputing 123, 207-215 (2014)

48. Zhang, ZQ, Liu, KY: Existence and global exponential stability of a periodic solution to interval general bidirectional associative memory (BAM) neural networks with multiple delays on time scales. Neural Netw. 24, 427-439 (2011)

49. Li, YK, Zhang, TW: Global exponential stability of fuzzy interval delayed neural networks with impulses on time scales. Int. J. Neural Syst. 19(6), 449-456 (2009)

50. Li, YK, Gao, S: Global exponential stability for impulsive BAM neural networks with distributed delays on time scales. Neural Process. Lett. 31, 65-91 (2010)

51. Bohner, M, Peterson, A: Advances in Dynamic Equations on Time Scales. Birkhäuser, Boston (2003)

52. Li, YK, Wang, C: Uniformly almost periodic functions and almost periodic solutions to dynamic equations on time scales. Abstr. Appl. Anal. 2011, Article ID 341520 (2011)

53. Li, YK, Wang, C: Almost periodic functions on time scales and applications. Discrete Dyn. Nat. Soc. 2011, Article ID $727068(2011)$

doi:10.1186/1687-1847-2014-178

Cite this article as: Li et al.: Almost periodic solutions for neutral delay Hopfield neural networks with time-varying delays in the leakage term on time scales. Advances in Difference Equations 2014 2014:178. 